\title{
40. PETROGRAPHY AND GEOCHEMISTRY OF VOLCANIC GLASS: LEG 57, DEEP SEA DRILLING PROJECT
}

\author{
Kantaro Fujioka and Toshio Furuta, Ocean Research Institute, University of Tokyo, Nakano, Tokyo Japan \\ and \\ Fusao Arai, Department of Geology, Gunma University, Maebashi, Japan
}

\begin{abstract}
About 200 volcanic ash layers were recovered during DSDP Leg 57. The volcanic glass in some of these layers was investigated petrographically and chemically in this study. Volcanic glass is mainly rhyolitic and/or rhyodacitic in chemical composition, and its refractive index ranges from 1.496 to 1.529 . Some volcanic ash layers consist of multiple grains of different chemical compositions. All the volcanic glass belongs to the tholeiitic and the calc-alkalic volcanic rock series, in $\mathrm{SiO}_{2}-\left(\mathrm{Na}_{2} \mathrm{O}+\mathrm{K}_{2} \mathrm{O}\right)$ diagram and $\mathrm{FeO} * / \mathrm{MgO}-\mathrm{SiO}_{2}$ diagram. We correlated successfully three volcanic ash layers from the standpoint of chemical composition and biostratigraphy. Hydration of volcanic glass from Leg 57 is less intense than in other DSDP cores.
\end{abstract}

\section{INTRODUCTION}

Volcanic glass occurs in distinct ash layers, small pods or pockets, and as dispersed minor constituents of sediments and sedimentary rocks in the cores of DSDP Leg 57. They range in age from lower Miocene to Recent and differ from one another in various respects. Chemical composition is mainly rhyolitic and/or rhyodacitic, dacitic, and, infrequently, basaltic. Grains are typically silt-sized.

Investigation of volcanic ash has been very fruitful and provides a great deal of information about the volcanicity of source regions, chemical composition of a single volcano, hydration under conditions of burial, and so on. Because the study of volcanic ash is only beginning, many problems remain unsolved. In the present chapter we present fundamental data on volcanic ash: description, grain-size measurements, type of glass, mineralogy and chemical composition of glass shards, as well as a short preliminary discussion of original magma and alteration.

\section{DESCRIPTION OF VOLCANIC GLASS}

\section{Visual Description}

During Leg 57 more than 200 volcanic ash layers were identified by visual observation of the cores. A small number of ashy pods or pockets and minor amounts of dispersed volcanic glass were also distinguished in sediments and sedimentary rocks. The ash derives chiefly from the nearby Japanese Islands, transported by prevailing westerlies. Active Quaternary volcanoes, distributed west of the volcanic front of Honshu-the main land mass-supply a tremendous amount of volcanic ash to the area of the Japan Trench. Machida and Arai (1976) have summarized the distribution of typical Quaternary Japanese tephras, mapping several tephras which might have been able to reach Leg 57 sites. For example, the distribution of the tephra To-HP (Hachinohe pumice of Mt. Towada) and $\mathrm{Spfa}_{1}$ (Mt. Shikotsu pumice fall) have a local distribution pattern large enough to include all sites. It is possible to identify both of these tephras at Leg 57 sites.

In the Pliocene and Miocene volcanic activity in the Japanese Islands was intense, judging from the volcanic and pyroclastic rocks and from the dispersed constituents in the sediments on land. All the volcanic ash layers and ashy pods or pockets obtained during Leg 57 are described in Table 1, with short remarks on each layer observed onboard. Ashes in Hole 436, Leg 56, are also listed in the table. At Sites 438 and 439 at the deep sea terrace, 132 distinct ash layers were distinguished throughout the recovered sedimentary column. The thickest ash layer occurs at Site 438, Core 438-11,CC and is over $16 \mathrm{~cm}$. However, layers are usually less than $5 \mathrm{~cm}$ thick.

At Site 440 , on a sedimentary pond at the trench slope bench, 57 ash layers were identified, the thickest of which is $10 \mathrm{~cm}$. The difference in number of ash layers observed at Sites 438 and 439 and at Site 440 depends chiefly on time of deposition, even though Sites 438 and 439 are closer to the continent. Sites 438 and 439 penetrate lower Miocene and Site 440, upper Miocene. At Site 436 , on the Pacific ocean floor, 57 ash layers were identified. Both Sites 440 and 436 are similar in number of ash layers and in sediment age. Debates concerning methods of estimating the volcanicity of regions are common. Kennett and Thunell (1975) demonstrated that the volcanic ash in deep sea sediments indicate very high rates of explosive volcanism during the last two million years. Ninkovich and Donn (1976) and Hein and Scholl (1978) have proposed a model based upon movement of the oceanic plate. Because of drilling disturbance, bioturbation, recovery rate, and many other factors, it is very difficult to record all the volcanicity in 
TABLE 1

Volcanic Ash, DSDP Leg 57

\begin{tabular}{|c|c|c|c|c|c|c|c|c|}
\hline $\begin{array}{c}\text { Sample } \\
\text { (Interval in } \mathrm{cm} \text { ) }\end{array}$ & $\begin{array}{l}\text { Thickness } \\
(\mathrm{cm})\end{array}$ & $\begin{array}{l}\text { Smear Slide } \\
\text { (cm depth) }\end{array}$ & Layer Number & Remarks & Age & $\underset{(\%)}{\mathrm{SiO}_{2}}$ & $\begin{array}{c}\Sigma \mathrm{Fe}_{2} \mathrm{O}_{3} \\
(\%)\end{array}$ & Index \\
\hline \multicolumn{9}{|l|}{ Hole 438} \\
\hline $\begin{array}{l}1-1,62-67 \\
1-1,89-100 \\
1-2,10-15 \\
1-2,20-24 \\
1-2,68-73\end{array}$ & $\begin{array}{r}5 \\
11 \\
5 \\
4 \\
5\end{array}$ & & & $\begin{array}{l}\text { pod, coarse pumiceous sand } \\
\text { pod, coarse pumiceous sand } \\
\text { pod, } 5 Y 4 / 1 \text {, coarse pumiceous sand } \\
\text { pod, } 5 Y 4 / 1 \text {, coarse pumiceous sand } \\
\text { pod, } 5 \text { Y } 4 / 1 \text {, coarse pumiceous sand }\end{array}$ & upper Pleistocene & & & \\
\hline $\begin{array}{l}2-3,102-105 \\
2-3,128-132 \\
2-4,122-125 \\
2-5,21-32 \\
2-5,56-57\end{array}$ & $\begin{array}{r}3 \\
4 \\
3 \\
11 \\
1\end{array}$ & 123 & 001 & $\begin{array}{l}\text { pod, white-light gray } \\
\text { pocket } \\
\text { pod, white gray } \\
\text { layer, multiple sandy, light gray } \\
\text { pocket, small }\end{array}$ & & & & \\
\hline $\begin{array}{l}2-5,106-111 \\
2-6,24-26 \\
2, C C, 5-6 \\
3-1,86-88 \\
3-2,15-26\end{array}$ & $\begin{array}{r}5 \\
2 \\
1 \\
2 \\
11\end{array}$ & 110 & 002 & $\begin{array}{l}\text { layer, light gray-white } \\
\text { pod } \\
\text { pocket, gray-white } \\
\text { pocket } \\
\text { pocket, pumiceous }\end{array}$ & & 76.45 & 2.06 & $1.502-1.506$ \\
\hline $\begin{array}{l}3-6,91-98 \\
7-2,48-50 \\
7-2,103-105 \\
7-7,2-5 \\
8-3,2-4\end{array}$ & $\begin{array}{l}7 \\
2 \\
2 \\
3 \\
2\end{array}$ & $\begin{array}{r}96 \\
49 \\
104 \\
3\end{array}$ & $\begin{array}{l}003 \\
004 \\
005 \\
006\end{array}$ & $\begin{array}{l}\text { layer, gray-white } \\
\text { layer, } 5 \mathrm{~B} 6 / 1 \text {, medium-grained } \\
\text { layer, } 5 \mathrm{~B} 6 / 1 \text {, medium-grained } \\
\text { layer, } 5 \mathrm{Y} 3 / 2 \text {, medium-grained } \\
\text { pocket, black }\end{array}$ & upper Pliocene & 75.48 & 1.47 & $1.500-1.506$ \\
\hline $\begin{array}{l}8-3,3-7 \\
8-3,7-11 \\
8-4,43-49 \\
8-4,70-73 \\
8-4,74-83\end{array}$ & $\begin{array}{l}4 \\
4 \\
6 \\
3 \\
9\end{array}$ & & & $\begin{array}{l}\text { pocket, white } \\
\text { pocket, white } \\
\text { pocket, black } \\
\text { pocket, black } \\
\text { pocket, black }\end{array}$ & & & & \\
\hline $\begin{array}{l}8-4,81-82 \\
8-4,83-86 \\
8-4,95-97 \\
8-4,100-104 \\
8-4,114-117\end{array}$ & $\begin{array}{l}1 \\
3 \\
2 \\
4 \\
3\end{array}$ & & & $\begin{array}{l}\text { pocket, small } \\
\text { pocket } \\
\text { pocket, small } \\
\text { pocket } \\
\text { pocket }\end{array}$ & & & & \\
\hline $\begin{array}{l}8-5,11-15 \\
8-7,9-12 \\
10-4,17-24 \\
10-5,17-22 \\
10-5,81-83\end{array}$ & $\begin{array}{l}4 \\
3 \\
7 \\
5 \\
2\end{array}$ & $\begin{array}{l}23 \\
82\end{array}$ & $\begin{array}{l}007 \\
008 \\
009 \\
010\end{array}$ & $\begin{array}{l}\text { pocket } \\
\text { layer, N6-N } 4 \text {, fine-grained, gray } \\
\text { layer, } 5 \text { Y } 5 / 1 \text {, fine-grained, gray } \\
\text { layer, } 5 \text { Y } 3 / 1 \text {, fine-grained, gray } \\
\text { layer, N5-N3, fine-grained }\end{array}$ & & 78.21 & $\begin{array}{l}1.48 \\
1.37\end{array}$ & \\
\hline $\begin{array}{l}10-6,20-25 \\
10-6,25-33 \\
11-1,9-16 \\
11-1,31-36 \\
11-1,41-45\end{array}$ & $\begin{array}{l}5 \\
8 \\
7 \\
5 \\
4\end{array}$ & $\begin{array}{l}23 \\
27\end{array}$ & $\begin{array}{l}011 \\
012 \\
013 \\
014 \\
015\end{array}$ & $\begin{array}{l}\text { layer, } 5 B 7 / 1 \text {, silty } \\
\text { layer, } 5 Y 3 / 1 \text {, fine } \\
\text { layer, } 5 Y 2 / 1 \text {, fine } \\
\text { layer, } N 4-N 5 \text {, medium-grained } \\
\text { layer, } N 4 \text {, medium-grained }\end{array}$ & & & & \\
\hline $\begin{array}{l}11-3,29-36 \\
11-3,88-94 \\
11-3,125-131 \\
11-4,58-65 \\
11-4,83-95\end{array}$ & $\begin{array}{r}7 \\
6 \\
6 \\
7 \\
12\end{array}$ & & $\begin{array}{l}016 \\
017 \\
018 \\
019 \\
020\end{array}$ & $\begin{array}{l}\text { layer, } \mathrm{N} 4 \text {, coarse-grained } \\
\text { layer, } \mathrm{N} 4 \text {, sandy } \\
\text { layer, } 5 \mathrm{~B} 6 / 1-5 \mathrm{Y} 3 / 1 \text {, sandy } \\
\text { layer, } 5 \mathrm{~B} 6 / 1 \text {, medium-grained } \\
\text { layer, } 5 \mathrm{~B} 6 / 1 \text {, medium-grained }\end{array}$ & & & & $1.506-1.508$ \\
\hline $\begin{array}{l}11-4,111-115 \\
11-5,26-32 \\
11-5,106-111 \\
11, C C, 4-20 \\
12-5,60-61\end{array}$ & $\begin{array}{r}4 \\
6 \\
5 \\
16 \\
1\end{array}$ & 61 & $\begin{array}{l}021 \\
022 \\
023 \\
024\end{array}$ & $\begin{array}{l}\text { layer, } 5 \mathrm{~B} 6 / 1 \text {, medium-grained } \\
\text { layer, } 5 \mathrm{~B} 6 / 1 \text {, medium-grained } \\
\text { layer, } 5 \mathrm{~B} 7 / 1 \text {, medium-grained } \\
\text { layer, } 5 \mathrm{~B} 6 / 1 \text {, silty } \\
\text { layer, ashy sand }\end{array}$ & & & & \\
\hline \multicolumn{9}{|l|}{ Hole 438A } \\
\hline $\begin{array}{l}2-2,104-106 \\
2-2,122-129 \\
2-3,24-27 \\
2-3,51-55 \\
2-4,119-120\end{array}$ & $\begin{array}{l}2 \\
7 \\
3 \\
4 \\
1\end{array}$ & 124 & $\begin{array}{l}025 \\
026\end{array}$ & $\begin{array}{l}\text { pocket } \\
\text { layer, } 5 \mathrm{G} 4 / 1 \\
\text { pocket, } 5 \mathrm{G} 4 / 1 \\
\text { pocket } \\
\text { layer }\end{array}$ & upper Pleistocene & & & \\
\hline $\begin{array}{l}2-4,130-134 \\
2-5,15-23 \\
2-5,32-35 \\
2-5,42-48 \\
2-6,105-110\end{array}$ & $\begin{array}{l}4 \\
8 \\
3 \\
6 \\
5\end{array}$ & 21 & $\begin{array}{l}027 \\
028\end{array}$ & $\begin{array}{l}\text { layer, } 5 \mathrm{G} 4 / 1 \\
\text { layer, } 5 \mathrm{~B} 7 / 1 \text {, silty } \\
\text { pocket } \\
\text { pocket } \\
\text { pocket, } 5 \mathrm{G} 4 / 1\end{array}$ & & 78.41 & 1.19 & $1.502-1.503$ \\
\hline $\begin{array}{l}3-2,116-125 \\
3-2,148-150 \\
3-5,94-106 \\
3-6,97-100 \\
3-6,102-104\end{array}$ & $\begin{array}{r}9 \\
2 \\
12 \\
3 \\
2\end{array}$ & 100 & $\begin{array}{l}029 \\
030 \\
031 \\
032 \\
033\end{array}$ & $\begin{array}{l}\text { layer, } 5 G 4 / 1 \text {, coarse sandy } \\
\text { layer, coarse sandy } \\
\text { layer, } 5 G Y 4 / 1 \text {, sandy } \\
\text { layer, } 5 G 4 / 1 \text {, very fine grained } \\
\text { layer, very fine grained }\end{array}$ & lower Pleistocene & 75.48 & 3.79 & $1.496-1.498$ \\
\hline $\begin{array}{l}3-6,117-120 \\
4-3,129-134 \\
4-3,141-147 \\
4-4,12-14 \\
4-4,38-40\end{array}$ & $\begin{array}{l}3 \\
5 \\
6 \\
2 \\
2\end{array}$ & & $\begin{array}{l}034 \\
035\end{array}$ & $\begin{array}{l}\text { layer, } 5 G Y 4 / 1 \text {, dark gray } \\
\text { layer, inclined, } 5 G Y 4 / 1 \text {, silty } \\
\text { pocket } \\
\text { pocket, small, } 5 Y 4 / 1 \\
\text { pod, silt-sized }\end{array}$ & $\begin{array}{l}\text { lower Pleistocene } \\
\text { upper Pliocene }\end{array}$ & & & \\
\hline $\begin{array}{l}4-4,72-76 \\
4-6,81-84 \\
4-6,100-105 \\
4-6,113-115 \\
5-3,77-85\end{array}$ & $\begin{array}{l}4 \\
3 \\
5 \\
2 \\
8\end{array}$ & 78 & $\begin{array}{l}036 \\
037\end{array}$ & $\begin{array}{l}\text { pocket } \\
\text { small pocket } \\
\text { layer, arched, ver y fine-grained, } 5 \mathrm{GY} 4 / 1 \\
\text { pocket } \\
\text { layer, } 5 \mathrm{G} 2 / 1 \text {, tine-grained }\end{array}$ & upper Pliocene & & & \\
\hline
\end{tabular}


TABLE 1 - Continued

\begin{tabular}{|c|c|c|c|c|c|c|c|c|}
\hline $\begin{array}{c}\text { Sample } \\
\text { (Interval in } \mathrm{cm} \text { ) }\end{array}$ & $\begin{array}{l}\text { Thickness } \\
(\mathrm{cm})\end{array}$ & $\begin{array}{l}\text { Smear Slide } \\
\text { (cm depth) }\end{array}$ & Layer Number & Remarks & Age & $\begin{array}{l}\mathrm{SiO}_{2} \\
(\%)\end{array}$ & $\underset{(\%)}{\mathrm{\Sigma Fe}_{2} \mathrm{O}_{3}}$ & Index \\
\hline \multicolumn{9}{|l|}{ Hole 438A (cont.) } \\
\hline $\begin{array}{l}5-3,103-104 \\
6-2,71-74 \\
6-3,72-73 \\
6-3,85-86 \\
6-3,123-127\end{array}$ & $\begin{array}{l}1 \\
3 \\
1 \\
1 \\
4\end{array}$ & 74 & $\begin{array}{l}038 \\
039 \\
040 \\
041\end{array}$ & $\begin{array}{l}\text { pocket, small } \\
\text { layer, } 5 G Y 2 / 1 \text {, silty } \\
\text { layer, } 5 G 4 / 1 \text {, very fine grained } \\
\text { layer, } 5 B 5 / 1 \text {, medium-grained } \\
\text { layer, } 5 B 5 / 1 \text {, silty, graded }\end{array}$ & & & & \\
\hline $\begin{array}{l}6-3,148-150 \\
6-4,17-24 \\
6-4,72-73 \\
7-4,51-55 \\
7-4,71-75\end{array}$ & $\begin{array}{l}2 \\
7 \\
1 \\
4 \\
4\end{array}$ & & $\begin{array}{l}042 \\
043 \\
\\
044 \\
045\end{array}$ & $\begin{array}{l}\text { layer, } 5 B 5 / 1 \text {, very fine grained } \\
\text { layer, } 5 B 5 / 1 \text {, medium-grained } \\
\text { pocket, very fine grained } \\
\text { layer, } 5 Y 2.5 / 1 \text {, sandy } \\
\text { layer, } 5 Y 7 / 1 \text {, very fine grained }\end{array}$ & & & & $1.498-1.499$ \\
\hline $\begin{array}{l}7-5,45-51 \\
8-4,98-100 \\
8-3,95 \\
8-5,69-70 \\
10-3,86-88\end{array}$ & $\begin{array}{r}6 \\
2 \\
>1 \\
1 \\
2\end{array}$ & $\begin{array}{r}50 \\
100 \\
\\
87\end{array}$ & $\begin{array}{l}046 \\
047 \\
048 \\
049\end{array}$ & $\begin{array}{l}\text { layer, } 2.5 \mathrm{YN} 4 / \text {, sandy } \\
\text { layer, } 2.5 \mathrm{YN} 6 \% \text {, fine-grained } \\
\text { layer, } 2.5 \mathrm{YN} 4 / 1 \text {, medium coarse grained } \\
\text { layer, medium-grained } \\
\text { pod, } 2.5 \mathrm{YN} 2.5 \%\end{array}$ & & & & \\
\hline $\begin{array}{l}11-2,81-83 \\
11-4,35-40 \\
11-6,0-2 \\
11-6,103-105 \\
12-1,65-66\end{array}$ & $\begin{array}{l}2 \\
5 \\
2 \\
1\end{array}$ & & $\begin{array}{l}050 \\
051\end{array}$ & $\begin{array}{l}\text { pocket, } 2.5 \mathrm{YN} 2.5 \text { / } \\
\text { layer, } 2.5 \mathrm{YN} 6 / \text {, medium-grained } \\
\text { layer, } 5 \text { Y } 4 / 2 \\
\text { pod, small, } 2.5 \mathrm{YN} 3 / \\
\text { pocket, medium-grained, } 2.5 \mathrm{YN} 3 /\end{array}$ & $\begin{array}{l}\text { upper Pliocene } \\
\text { lower Pliocene }\end{array}$ & & & \\
\hline $\begin{array}{l}12-1,70-71 \\
12-1,73-74 \\
12-1,87-88 \\
12-3,16-19 \\
13-3,35-37\end{array}$ & $\begin{array}{l}1 \\
1 \\
1 \\
3 \\
2\end{array}$ & & $\begin{array}{l}052 \\
053\end{array}$ & $\begin{array}{l}\text { pocket, medium-grained } \\
\text { pocket, medium-grained } \\
\text { pocket, } 2.5 \mathrm{YN} 7 / \text {, coarse-grained } \\
\text { layer, } 2.5 \mathrm{YN} 8 / \text {, medium-grained } \\
\text { layer, } 2.5 \mathrm{YN} 3 / \text {, medium coarse grained }\end{array}$ & lower Pliocene & & & \\
\hline $\begin{array}{l}13-5,19-24 \\
14-2,11-14 \\
14-2,18-22 \\
14-2,103-108 \\
14-3,7-8\end{array}$ & $\begin{array}{l}5 \\
3 \\
4 \\
5 \\
1\end{array}$ & & $\begin{array}{l}054 \\
055 \\
056 \\
057\end{array}$ & $\begin{array}{l}\text { layer, } 2.5 \mathrm{YN} 8 / \text {, medium-grained } \\
\text { layer, } 5 \text { Y } 8 / 1 \\
\text { layer, inclined, } 5 \text { Y } 6 / 1 \\
\text { pocket, } 7.5 \mathrm{YN} 6 / \text {, fine-grained } \\
\text { layer, fine-grained }\end{array}$ & lower Pliocene & & & \\
\hline $\begin{array}{l}14-3,127 \\
14-4,42-45 \\
14-4,87-88 \\
14-4,88-90 \\
14-4,117-120\end{array}$ & $\begin{array}{r}>1 \\
3 \\
1 \\
2 \\
3\end{array}$ & & $\begin{array}{l}058 \\
059 \\
060 \\
061 \\
062\end{array}$ & $\begin{array}{l}\text { layer, fine-grained } \\
\text { layer, } 2.5 \mathrm{YN} 4 / \text {, coarse pumiceous } \\
\text { layer, } 5 \mathrm{Y} 7 / 1 \text {, fine-grained pumiceous } \\
\text { layer, } 2.5 \mathrm{YN} 4 / \text {, medium-grained pumiceous } \\
\text { layer, } 2.5 \mathrm{YN} 8 / \text {, medium-grained }\end{array}$ & & & & \\
\hline $\begin{array}{l}16-3,142-147 \\
16-4,0-26 \\
16-5,86 \\
16, C C, 10 \\
17-1,72-75\end{array}$ & $\begin{array}{r}5 \\
26 \\
>1 \\
>1 \\
3\end{array}$ & 74 & $\begin{array}{l}063 \\
064 \\
065 \\
066\end{array}$ & $\begin{array}{l}\text { layer, inclined, } 2.5 \mathrm{YN} 6 / \text {, medium-grained } \\
\text { pod, thin, elongated downward } \\
\text { layer, fine-grained, } 2.5 \mathrm{YN} 6 / \\
\text { layer, } 5 \mathrm{Y} 7 / 1 \text {, fine-grained pumiceous } \\
\text { layer, medium-grained }\end{array}$ & & & & $1.501-1.503$ \\
\hline $\begin{array}{l}17-1,115-116 \\
17-1,117-119 \\
17-2,145-147 \\
17-2,147-149 \\
17-3,52-54\end{array}$ & $\begin{array}{l}1 \\
2 \\
2 \\
2 \\
2\end{array}$ & & $\begin{array}{l}067 \\
068\end{array}$ & $\begin{array}{l}\text { pocket, fine-grained, } 2.5 \text { YN } 7 / \\
\text { pocket, dark gray, silty } \\
\text { pod, very fine grained,gray } \\
\text { layer, fine-grained, dark gray } \\
\text { layer, } 2.5 \mathrm{YN} 9 /, \text { silty }\end{array}$ & & & & $1.508-1.511$ \\
\hline $\begin{array}{l}17-3,100-104 \\
18-2,35-40 \\
18-4,27-29 \\
18-4,90-100 \\
19-3,93-97\end{array}$ & $\begin{array}{r}4 \\
5 \\
2 \\
10 \\
4\end{array}$ & $\begin{array}{r}101 \\
39 \\
96\end{array}$ & $\begin{array}{l}069 \\
070 \\
071 \\
072 \\
073\end{array}$ & $\begin{array}{l}\text { layer, dark gray } \\
\text { layer, } 2.5 \mathrm{YN} 8 \% \text {, medium fine grained } \\
\text { layer, } 7.5 \mathrm{YN} 6 / \text {, medium-grained } \\
\text { layer, fine-grained, dark gray, } 7.5 \mathrm{YN} 3 \text { / } \\
\text { layer, N6., fine-grained }\end{array}$ & & & & \\
\hline $\begin{array}{l}19-3,102-105 \\
19-4,69-72 \\
19-5,31-35 \\
20-2,125-126 \\
20-2,135-137\end{array}$ & $\begin{array}{l}3 \\
3 \\
4 \\
1 \\
2\end{array}$ & 103 & $\begin{array}{l}074 \\
075 \\
076 \\
077 \\
078\end{array}$ & $\begin{array}{l}\text { layer, } 5 Y 3 / 2 \text {, medium-grained, silty } \\
\text { layer, N7, fine-grained } \\
\text { layer, N5, very fine grained } \\
\text { layer, N5, very fine grained } \\
\text { layer, N5, fine-grained }\end{array}$ & & & & \\
\hline $\begin{array}{l}21-1,6-12 \\
21-6,87-88 \\
24-1,52-55 \\
24-1,97-101 \\
24-1,125-130\end{array}$ & $\begin{array}{l}6 \\
1 \\
3 \\
4 \\
5\end{array}$ & $\begin{array}{r}9 \\
127\end{array}$ & $\begin{array}{l}079 \\
080 \\
081 \\
082 \\
083\end{array}$ & $\begin{array}{l}\text { layer, N6, very fine grained } \\
\text { layer, N5, very fine grained } \\
\text { layer, SY3/2, fine-grained, silty } \\
\text { layer, N5, very fine grained } \\
\text { layer, N4, very fine grained }\end{array}$ & & & & \\
\hline $\begin{array}{l}24-1,148-150 \\
24-2,0-7 \\
24-3,80-82 \\
24-4,57-61 \\
24-6,83-87\end{array}$ & $\begin{array}{l}2 \\
7 \\
2 \\
4 \\
4\end{array}$ & 85 & $\begin{array}{l}084 \\
084 \\
085 \\
086 \\
087\end{array}$ & $\begin{array}{l}\text { layer, sandy, } 5 Y 3 / 2 \\
\text { layer, sandy, } 5 \text { Y } 3 / 2 \\
\text { layer, } \mathrm{N} 4 \\
\text { layer, } 5 \mathrm{~B} 8 / 1 \text {, silt-sized } \\
\text { layer, } 5 \mathrm{Y} 6 / 1 \text {, very fine grained }\end{array}$ & lower Pliocene & & & \\
\hline $\begin{array}{l}25-3,34-38 \\
25-4,34-38 \\
26-2,11-14 \\
27-3,81-83 \\
27-6,81-88\end{array}$ & $\begin{array}{l}4 \\
4 \\
3 \\
2 \\
7\end{array}$ & $\begin{array}{l}35 \\
11 \\
82 \\
85\end{array}$ & $\begin{array}{l}088 \\
089 \\
090 \\
091 \\
092\end{array}$ & $\begin{array}{l}\text { layer, } N 7 \text {, very fine grained } \\
\text { layer, } 5 Y 4 / 1 \text {, silty } \\
\text { layer, N7, silt-sized } \\
\text { layer, dark gray, medium-grained sandy } \\
\text { layer, light gray, graded }\end{array}$ & & & & $1.501-1.503$ \\
\hline $\begin{array}{l}28-4,62-65 \\
28-4,141-142 \\
29-5,90-92 \\
30-4,34-35 \\
30-7,19-21\end{array}$ & $\begin{array}{l}3 \\
1 \\
2 \\
1 \\
2\end{array}$ & & $\begin{array}{l}093 \\
094\end{array}$ & $\begin{array}{l}\text { pod, dark gray, silty } \\
\text { layer, fine-grained, silty, light gray graded } \\
\text { layer, gray, N.7 } \\
\text { pocket, light gray } \\
\text { pocket, light gray-white }\end{array}$ & & & & \\
\hline $\begin{array}{l}31-5,60-62 \\
32-1,98-100\end{array}$ & $\begin{array}{l}2 \\
2\end{array}$ & 61 & $\begin{array}{l}095 \\
096\end{array}$ & $\begin{array}{l}\text { layer, silty, 10GY5/2 } \\
\text { layer, sand-sized, light gray }\end{array}$ & & & & \\
\hline
\end{tabular}


TABLE 1 - Continued

\begin{tabular}{|c|c|c|c|c|c|c|c|c|}
\hline $\begin{array}{c}\text { Sample } \\
\text { (Interval in } \mathrm{cm} \text { ) }\end{array}$ & $\begin{array}{l}\text { Thickness } \\
\text { (cm) }\end{array}$ & $\begin{array}{l}\text { Smear Slide } \\
\text { (cm depth) }\end{array}$ & Layer Number & Remarks & Age & $\underset{(\%)}{\mathrm{SiO}_{2}}$ & $\begin{array}{c}\mathrm{\Sigma Fe}_{2} \mathrm{O}_{3} \\
(\%)\end{array}$ & Index \\
\hline \multicolumn{9}{|l|}{ Hole $438 \mathrm{~A}$ (cont.) } \\
\hline $\begin{array}{l}32-1,141-142 \\
32-1,143-144 \\
32-1,145-146\end{array}$ & $\begin{array}{l}1 \\
1 \\
1\end{array}$ & & & $\begin{array}{l}\text { pocket, irregular } \\
\text { pocket, very fine grained } \\
\text { pocket, dark gray }\end{array}$ & & & & \\
\hline $\begin{array}{l}32-2,15-18 \\
32-3,90-93 \\
33-4,74-75 \\
33-5,141-143 \\
33-6,128-133\end{array}$ & $\begin{array}{l}3 \\
3 \\
1 \\
2 \\
5\end{array}$ & $\begin{array}{l}91 \\
74\end{array}$ & $\begin{array}{l}097 \\
098\end{array}$ & $\begin{array}{l}\text { pocket, dark gray, medium fine grained } \\
\text { layer, medium fine grained, light gray, N5 } \\
\text { layer, light gray, N7 } \\
\text { pod, mottled, light gray } \\
\text { pocket, pumiceous }\end{array}$ & & & & \\
\hline $\begin{array}{l}34-3,84-87 \\
34-4,22-24 \\
35-3,28-30 \\
35-3,62-64 \\
35-3,108-111\end{array}$ & $\begin{array}{l}3 \\
2 \\
2 \\
2 \\
3\end{array}$ & $\begin{array}{r}29 \\
110\end{array}$ & $\begin{array}{l}099 \\
100 \\
101 \\
102\end{array}$ & $\begin{array}{l}\text { pocket, light gray } \\
\text { layer, silty, N5 } \\
\text { layer, gray, fine-grained } \\
\text { layer, light gray, very fine grained } \\
\text { layer, } 5 \text { Y } 4 / 2\end{array}$ & & & & \\
\hline $\begin{array}{l}35-6,102-111 \\
36-1,111-112 \\
36-5,67-71 \\
36-6,8-11 \\
36-6,57-59\end{array}$ & $\begin{array}{l}9 \\
1 \\
4 \\
3 \\
2\end{array}$ & 57 & $\begin{array}{l}103 \\
104 \\
105 \\
106\end{array}$ & $\begin{array}{l}\text { layer, gray, silty, top is chalk } \\
\text { layer, light gray, fine-grained } \\
\text { pocket } \\
\text { layer, fine-grained sandy } \\
\text { layer, fine to medium grained }\end{array}$ & upper Miocene & & & \\
\hline $\begin{array}{l}40-1,37-41 \\
40-2,9-11 \\
42-7,37-40 \\
43-2,83-85 \\
43-3,9-10\end{array}$ & $\begin{array}{l}4 \\
2 \\
3 \\
2 \\
1\end{array}$ & & $\begin{array}{l}107 \\
108\end{array}$ & $\begin{array}{l}\text { layer, } 5 \text { B5/1, gray, fine-grained } \\
\text { pocket, } 5 \mathrm{G} 4 / 1 \text {, fine-grained } \\
\text { layer, inclined, very fine grained, white } \\
\text { pocket, silty } \\
\text { pocket, } 5 Y 5 / 1 \text {, gray, silty }\end{array}$ & upper Miocene & & & \\
\hline $\begin{array}{l}43-3,83-87 \\
44-6,17-20 \\
45-4,5-10 \\
45-6,4-17 \\
46-5,5-8\end{array}$ & $\begin{array}{r}4 \\
3 \\
5 \\
13 \\
3\end{array}$ & 7 & $\begin{array}{l}109 \\
110 \\
111 \\
112\end{array}$ & $\begin{array}{l}\text { pocket, } 5 Y 5 / 1 \text {, gray } \\
\text { layer, } 5 \text { YR } 5 / 0, \text { dark gray } \\
\text { layer, wedge, } 5 \text { YR } 4 / 0, \text { silty } \\
\text { layer, inclined, } 5 \text { Y } 4 / 1 \text {, silty } \\
\text { layer, silty, N } 3 \text {, pyritized }\end{array}$ & & & & \\
\hline $\begin{array}{l}46-5,86-90 \\
50-1,33-35 \\
59-3,67-70 \\
60-1,71-73 \\
64-1,115-117\end{array}$ & $\begin{array}{l}4 \\
2 \\
3 \\
2 \\
2\end{array}$ & $\begin{array}{r}69 \\
71 \\
117\end{array}$ & $\begin{array}{l}113 \\
114\end{array}$ & $\begin{array}{l}\text { pocket, } 5 \text { YR } 4 / 0 \text {, dark gray, silty } \\
\text { layer } \\
\text { layer, sandy } \\
\text { pocket, pumiceous } \\
5 \mathrm{GY} 4 / 1 \text {, fine-grained, diatomaceous vitreous claystone }\end{array}$ & middle Miocene & & & \\
\hline $\begin{array}{l}64-3,27-32 \\
65-4,37-45 \\
65-4,105-112 \\
65-5,10-16 \\
71-5,120-125\end{array}$ & $\begin{array}{l}5 \\
8 \\
7 \\
6 \\
5\end{array}$ & $\begin{array}{r}30 \\
110 \\
13 \\
121\end{array}$ & $\begin{array}{l}115 \\
116 \\
117\end{array}$ & $\begin{array}{l}\text { layer, intercalated glauconite, silty } \\
\text { basalt, scoria, clast lappili } 5 \mathrm{~mm} \text {. } \\
\text { vitric diatomaceous claystone } \\
\text { layer, N7, silty } \\
\text { layer, very fine grained }\end{array}$ & & & & \\
\hline $\begin{array}{l}73-1,36-37 \\
79-6,85-86 \\
79-6,87-89 \\
84-2,85-86\end{array}$ & $\begin{array}{l}1 \\
1 \\
2 \\
1\end{array}$ & $\begin{array}{l}36 \\
86\end{array}$ & 118 & $\begin{array}{l}\text { layer, thin } \\
\text { pocket } \\
\text { pocket } \\
\text { pocket }\end{array}$ & & & & \\
\hline \multicolumn{9}{|l|}{ Hole 438B } \\
\hline $\begin{array}{l}6-2,63-66 \\
7-2,87-88 \\
11-2,3-4\end{array}$ & $\begin{array}{l}3 \\
1 \\
1\end{array}$ & 66 & & $\begin{array}{l}\text { pocket, } 5 B 5 / 1 \text {, medium-grained, gray } \\
\text { pocket } \\
\text { pocket, white, silt-sized }\end{array}$ & lower Miocene & & & \\
\hline \multicolumn{9}{|l|}{ Hole 439} \\
\hline $\begin{array}{l}5-1,119-120 \\
7-1,8-10 \\
7-1,120-125 \\
7-2,134-140 \\
8-2,123-127\end{array}$ & $\begin{array}{l}1 \\
2 \\
5 \\
6 \\
4\end{array}$ & 124 & $\begin{array}{l}119 \\
120 \\
121 \\
122\end{array}$ & $\begin{array}{l}\text { pod, mottled, silty } \\
\text { layer, inclined, N7, silt-sized } \\
\text { layer, inclined, N7, silty } \\
\text { layer, inclined, N6, very fine grained } \\
\text { layer, N6, very fine grained, cut by normal fault }\end{array}$ & $\begin{array}{l}\text { middle Miocene } \\
\text { lower Miocene }\end{array}$ & & & \\
\hline $\begin{array}{l}8-3,59-60 \\
8-3,63-65 \\
8-3,68-70 \\
8-3,78-80 \\
8-3,105-106\end{array}$ & $\begin{aligned}>1 \\
>1 \\
2 \\
2 \\
>1\end{aligned}$ & & $\begin{array}{l}123 \\
124 \\
125 \\
126 \\
127\end{array}$ & $\begin{array}{l}\text { layer, very fine grained } \\
\text { layer } \\
\text { layer, light gray } \\
\text { layer, silty } \\
\text { layer, light gray }\end{array}$ & lower Miocene & & & \\
\hline $\begin{array}{l}8-4,41-42 \\
8-5,16-17 \\
8-5,49-51 \\
8-5,98-100 \\
8-6,98-101\end{array}$ & $\begin{array}{l}1 \\
1 \\
2 \\
2 \\
3\end{array}$ & & 128 & $\begin{array}{l}\text { pocket, gray } \\
\text { pocket, white, fine-grained, silty } \\
\text { pocket, gray, sandy } \\
\text { layer, inclined } \\
\text { pocket, N5, medium-grained }\end{array}$ & & & & \\
\hline $\begin{array}{l}9-2,110-115 \\
9-3,61-65 \\
9-3,88-90 \\
9-3,102-110 \\
9-3,121-123\end{array}$ & $\begin{array}{l}5 \\
4 \\
2 \\
8 \\
2\end{array}$ & & $\begin{array}{l}129 \\
130\end{array}$ & $\begin{array}{l}\text { pocket, medium-grained, graded } \\
\text { pocket, } 3 \text { pieces, } 5 B 7 / 1 \\
\text { layer, } 5 B 5 / 1 \\
\text { layer, } 5 B 7 / 1 \text {, arched downward, silty } \\
\text { pocket, white, medium-grained }\end{array}$ & & & & $1.501-1.502$ \\
\hline $\begin{array}{l}9-5,38-52 \\
11-3,64-71 \\
13-1,0-17\end{array}$ & $\begin{array}{r}14 \\
7 \\
17\end{array}$ & & $\begin{array}{l}131 \\
132\end{array}$ & $\begin{array}{l}\text { layer, irregular upward } 5 B 5 / 1 \text {, graded } \\
\text { layer, } 5 B 5 / 1 \text {, irregular upward } \\
\text { tuffite }\end{array}$ & & & & \\
\hline \multicolumn{9}{|l|}{ Hole 440} \\
\hline $\begin{array}{l}4-1,104-112 \\
4-2,146-150 \\
4-3,38-48 \\
4-3,145-148 \\
4-6,130-134\end{array}$ & $\begin{array}{r}8 \\
4 \\
10 \\
3 \\
4\end{array}$ & 105 & $\begin{array}{l}001 \\
002 \\
003 \\
004\end{array}$ & $\begin{array}{l}\text { layer, N3, graded, base } 10 \mathrm{Y} 6 / 2 \\
\text { layer, graded, N3-10Y6/2 } \\
\text { pod } \\
\text { layer, arched upward, N4, mottled } \\
\text { layer, graded, N4(b), } 5 \mathrm{~B} 9 / 1(\mathrm{t})\end{array}$ & upper Pleistocene & & & \\
\hline
\end{tabular}


TABLE 1 - Continued

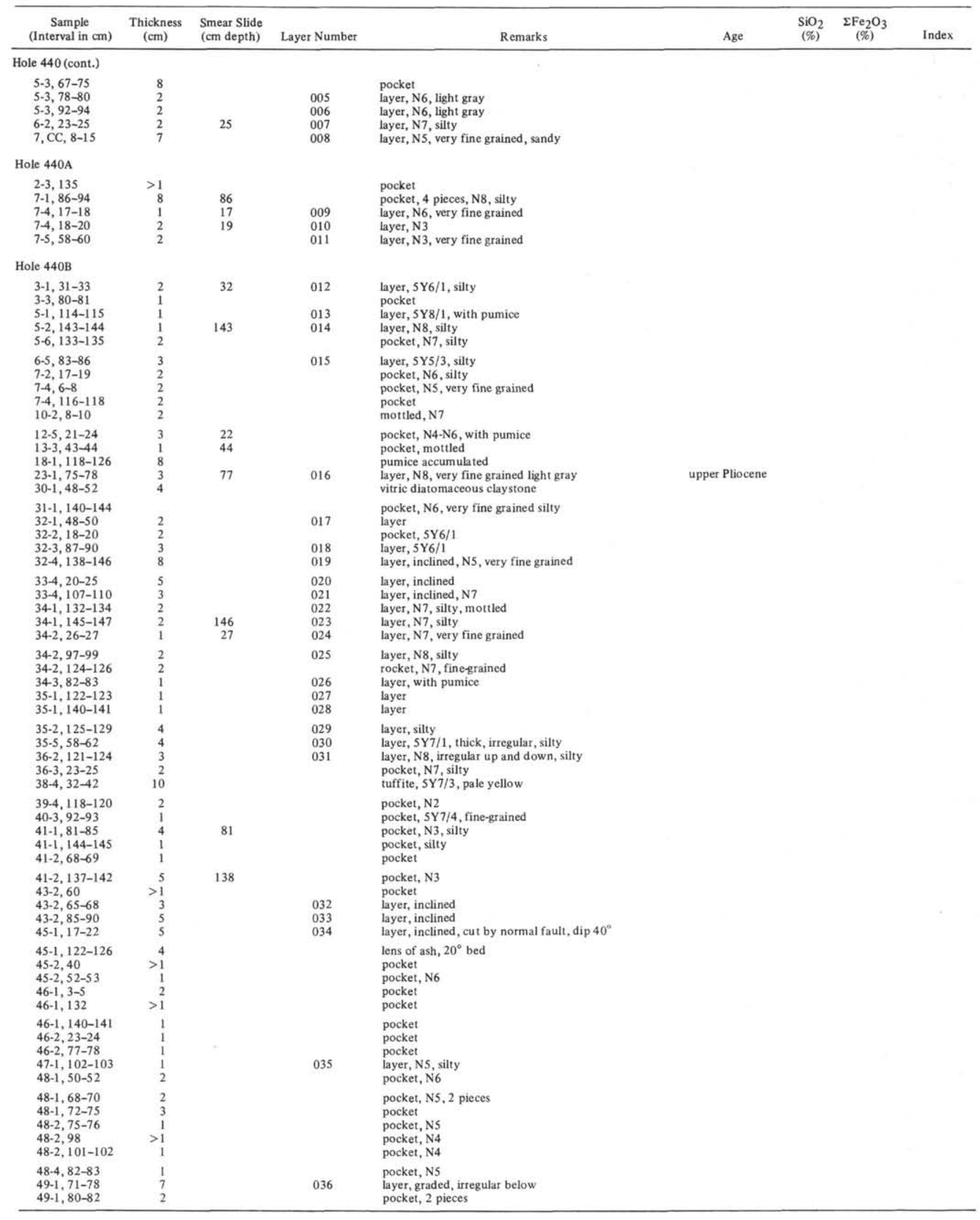


TABLE 1 - Continued

\begin{tabular}{|c|c|c|c|c|c|c|c|c|}
\hline $\begin{array}{c}\text { Sample } \\
\text { (Interval in } \mathrm{cm} \text { ) }\end{array}$ & $\begin{array}{l}\text { Thickness } \\
(\mathrm{cm})\end{array}$ & $\begin{array}{l}\text { Smear Slide } \\
\text { (cm depth) }\end{array}$ & Layer Number & Remarks & Age & $\begin{array}{l}\mathrm{SiO}_{2} \\
(\%)\end{array}$ & $\begin{array}{l}-\mathrm{Fe}_{2} \mathrm{O}_{3} \\
(\%)\end{array}$ & Index \\
\hline \multicolumn{9}{|l|}{ Hole $440 \mathrm{~B}$ (cont.) } \\
\hline $\begin{array}{l}49-2,77-87 \\
49-2,89-90\end{array}$ & $\begin{array}{c}10 \\
1\end{array}$ & & 037 & $\begin{array}{l}\text { layer, } 5 Y 5 / 2 \\
\text { pocket }\end{array}$ & & & & \\
\hline $\begin{array}{l}49-2,91-92 \\
49-2,97-100 \\
49-3,22-27 \\
49-3,30-32 \\
49-3,38\end{array}$ & $\begin{array}{r}1 \\
3 \\
5 \\
2 \\
>1\end{array}$ & & 038 & $\begin{array}{l}\text { pocket } \\
\text { pocket, N5 } \\
\text { layer, N5, irregular up and down } \\
\text { pocket, } 3 \text { pieces } \\
\text { pocket }\end{array}$ & & & & \\
\hline $\begin{array}{l}50-1,73-74 \\
50-1,135-137 \\
51-2,18-21 \\
51-2,87-88 \\
52-1,20-24\end{array}$ & $\begin{array}{l}1 \\
2 \\
3 \\
1 \\
4\end{array}$ & & 039 & $\begin{array}{l}\text { pocket, N5 } \\
\text { pocket, N5 } \\
\text { layer, N8, thin, mottled } \\
\text { mottled } \\
\text { pocket, N7 }\end{array}$ & & & & \\
\hline $\begin{array}{l}54-2,82-84 \\
55-3,44-47 \\
55-5,33-34 \\
55-5,36 \\
56-1,17\end{array}$ & $\begin{aligned} & 2 \\
& 3 \\
&> 1 \\
&> 1 \\
&> 1\end{aligned}$ & 36 & $\begin{array}{l}042 \\
043\end{array}$ & $\begin{array}{l}\text { pocket } \\
\text { pocket } \\
\text { layer, white calcareous } \\
\text { layer, thin } \\
\text { pocket }\end{array}$ & lower Pliocene & & & \\
\hline $\begin{array}{l}56-1,22 \\
56-1,48-50 \\
56-1,80 \\
56-2,44-51 \\
58-1,135-137\end{array}$ & $\begin{array}{r}>1 \\
2 \\
>1 \\
7 \\
2\end{array}$ & 80 & $\begin{array}{l}044 \\
045\end{array}$ & $\begin{array}{l}\text { pocket } \\
\text { layer, bioturbation } \\
\text { pocket } \\
\text { layer, } 5 \mathrm{GY} 6 / 1 \text {, tuffite } \\
\text { pocket, N6 }\end{array}$ & upper Miocene & & & \\
\hline $\begin{array}{l}58-5,134-135 \\
59-1,38-40 \\
59-3,86-88 \\
60-1,108-110 \\
60-2,53-55\end{array}$ & $\begin{array}{l}1 \\
2 \\
2 \\
2 \\
2\end{array}$ & & $\begin{array}{l}046 \\
047 \\
048 \\
049\end{array}$ & $\begin{array}{l}\text { layer, irregular } \\
\text { layer, N7 } \\
\text { layer, N5, arched downward } \\
\text { pocket, N7 } \\
\text { layer }\end{array}$ & & & & \\
\hline \multicolumn{9}{|l|}{ Hole 441} \\
\hline $\begin{array}{l}2-2,95-97 \\
2-4,55-57 \\
2-5,42-43 \\
2-5,77-82 \\
2-5,86-96 \\
7, C C, 19-20\end{array}$ & $\begin{array}{r}2 \\
2 \\
1 \\
5 \\
10 \\
1\end{array}$ & $\begin{array}{l}96 \\
42 \\
95\end{array}$ & & $\begin{array}{l}\text { pocket, } 5 \mathrm{Y} 5 / 3 \text {, clay-sized } \\
\text { pocket, } 5 \mathrm{Y} 4 / 3 \text {, silty } \\
\text { pocket, } 5 \mathrm{Y} 3 / 2 \text {, very fine grained } \\
\text { layer, } 5 \mathrm{Y} 7 / 1 \text {, silty } \\
\text { layer } 5 \mathrm{Y} 7 / 1, \text { silty } \\
\text { mottled, } \mathrm{N} 8 \text {, very fine grained silty }\end{array}$ & & & & \\
\hline \multicolumn{9}{|l|}{ Hole 441A } \\
\hline $\begin{array}{l}2-1,0-12 \\
11-1,70 \\
11-1,126 \\
H-1-3,99-101 \\
H-1-4,24-27 \\
H-1-4,48-51 \\
H-4-2,75-80\end{array}$ & $\begin{array}{r}>1 \\
>1 \\
2 \\
3 \\
3 \\
3 \\
5\end{array}$ & $\begin{array}{l}99 \\
25 \\
76\end{array}$ & & $\begin{array}{l}\text { tuffite, } 5 \text { Y } 4 / 1, \text { graded } \\
\text { pod } \\
\text { pod } \\
\text { layer, inclined, N8, mottled, dip } 15^{\circ} \\
\text { layer, inclined, silt-sized } \\
\text { pocket } \\
\text { layer, inclined, silty }\end{array}$ & & & & \\
\hline \multicolumn{9}{|l|}{ Hole 441B } \\
\hline $\begin{array}{l}1-1,83-99 \\
1-1,109-120 \\
\mathrm{H}-1-4,20-32\end{array}$ & $\begin{array}{l}16 \\
11 \\
12\end{array}$ & $\begin{array}{r}113 \\
23\end{array}$ & & $\begin{array}{l}\text { layer, } 5 Y 6 / 1, N 7 \text {, light olive } \\
\text { layer } \\
\text { pocket }\end{array}$ & & & & \\
\hline \multicolumn{9}{|l|}{ Hole 436} \\
\hline $\begin{array}{l}1-2,18-21 \\
1-2,82-85 \\
1-3,103-105 \\
1-4,20 \\
1-4,109-111\end{array}$ & $\begin{array}{r}3 \\
3 \\
2 \\
>1 \\
2\end{array}$ & $\begin{array}{r}21 \\
82 \\
105 \\
110\end{array}$ & $\begin{array}{l}001 \\
002\end{array}$ & $\begin{array}{l}\text { layer, N9-N7 } \\
\text { layer } \\
\text { pocket } \\
\text { pocket } \\
\text { pocket }\end{array}$ & Pleistocene & & & \\
\hline
\end{tabular}


TABLE 1 - Continued

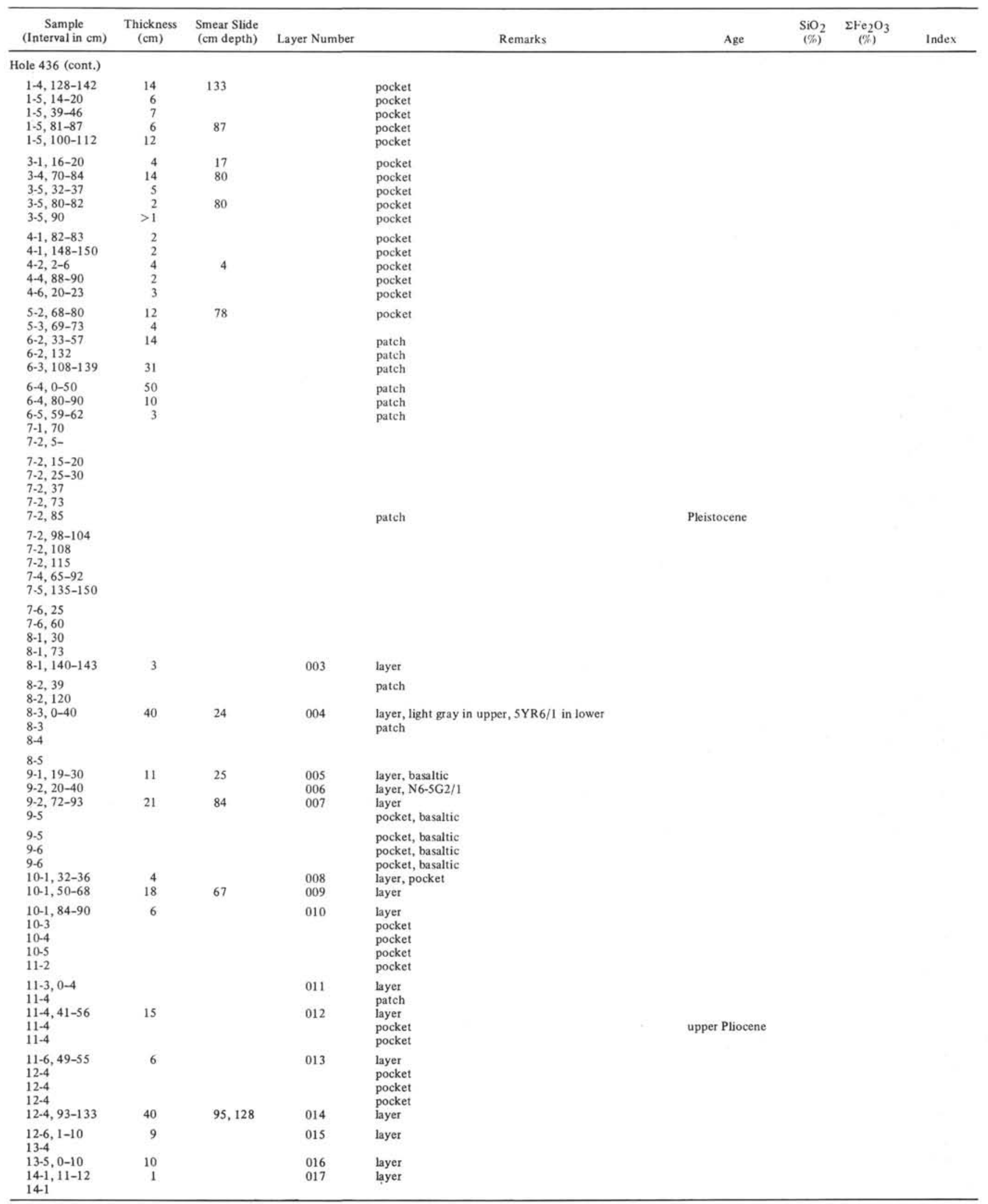


K. FUJIOKA, T. FURUTA, F. ARAI

TABLE 1 - Continued

\begin{tabular}{|c|c|c|c|c|c|c|c|c|c|}
\hline $\begin{array}{c}\text { Sample } \\
\text { (Interval in cm) }\end{array}$ & $\begin{array}{l}\text { Thickness } \\
(\mathrm{cm})\end{array}$ & $\begin{array}{l}\text { Smear Slide } \\
\text { (cm depth) }\end{array}$ & Layer Number & & Remarks & Age & $\begin{array}{c}\mathrm{SiO}_{2} \\
(\$)\end{array}$ & $\begin{array}{c}\Sigma \mathrm{F}_{2} \mathrm{O}_{3} \\
(\%)\end{array}$ & Index \\
\hline \multicolumn{10}{|l|}{ Hole 436 (cont.) } \\
\hline $\begin{array}{l}14-1 \\
14-2,51-60 \\
14-3 \\
14-3,60-66 \\
14-3,111-115\end{array}$ & $\begin{array}{l}9 \\
6 \\
4\end{array}$ & 58 & $\begin{array}{l}018 \\
019 \\
020\end{array}$ & $\begin{array}{l}\text { layer } \\
\text { layer } \\
\text { layer }\end{array}$ & & & & & \\
\hline $\begin{array}{l}14-4 \\
15-6,105-150 \\
15-7,0-10 \\
16-3,99-110 \\
16-4\end{array}$ & $\begin{array}{l}45 \\
10 \\
11\end{array}$ & $\begin{array}{l}115 \\
100\end{array}$ & $\begin{array}{l}021 \\
022 \\
023\end{array}$ & $\begin{array}{l}\text { layer, N7 } \\
\text { layer, N7 } \\
\text { layer, 5GY4/2 }\end{array}$ & & lower Pliocene & & & \\
\hline \multicolumn{10}{|l|}{$\begin{array}{l}16-4 \\
16-6 \\
16-6 \\
17-1 \\
17-1\end{array}$} \\
\hline $\begin{array}{l}17-3,50-60 \\
17-3 \\
17-3,99-110 \\
17-3 \\
17-3\end{array}$ & $\begin{array}{l}10 \\
11\end{array}$ & 55 & $\begin{array}{l}024 \\
025\end{array}$ & $\begin{array}{l}\text { layer } \\
\text { layer }\end{array}$ & & & & & \\
\hline $\begin{array}{l}17-4 \\
17, \mathrm{CC} \\
19-4,63-72 \\
20-1 \\
20-1,130-140\end{array}$ & $\begin{array}{r}9 \\
10\end{array}$ & 139 & $\begin{array}{l}026 \\
027\end{array}$ & $\begin{array}{l}\text { layer } \\
\text { layer }\end{array}$ & & lower Pliocene & & & \\
\hline $\begin{array}{l}20-2,15-25 \\
21-1\end{array}$ & 10 & 17 & 028 & layer & & & & & \\
\hline $\begin{array}{l}22-1,75-84 \\
22-1,112-117 \\
23-1,30-40\end{array}$ & $\begin{array}{r}9 \\
5 \\
10\end{array}$ & 80 & $\begin{array}{l}029 \\
030 \\
031\end{array}$ & $\begin{array}{l}\text { layer } \\
\text { layer } \\
\text { layer }\end{array}$ & & & & & \\
\hline $\begin{array}{l}23-1,47-50 \\
23-1,75-76 \\
23-2,10-16 \\
23-2,27-33 \\
23-2,70-73\end{array}$ & $\begin{array}{l}3 \\
1 \\
6 \\
6 \\
3\end{array}$ & & $\begin{array}{l}032 \\
033 \\
034 \\
035 \\
036\end{array}$ & $\begin{array}{l}\text { layer } \\
\text { layer } \\
\text { layer } \\
\text { layer } \\
\text { layer }\end{array}$ & & & & & \\
\hline $\begin{array}{l}23-2,100-102 \\
23-3,130-140 \\
23-4,20-26 \\
23-5\end{array}$ & $\begin{array}{r}2 \\
10 \\
6\end{array}$ & 138 & $\begin{array}{l}037 \\
038\end{array}$ & $\begin{array}{l}\text { layer } \\
\text { layer }\end{array}$ & & & & & \\
\hline $27-1,82-84$ & 2 & & 039 & layer & & upper Miocene & & & \\
\hline $\begin{array}{l}27-2,81-86 \\
27-3,6-10 \\
29-1,67-73 \\
29-1,112-120 \\
30-1,42-50\end{array}$ & $\begin{array}{l}5 \\
4 \\
6 \\
8 \\
8\end{array}$ & $\begin{array}{r}70 \\
118\end{array}$ & $\begin{array}{l}040 \\
041 \\
042 \\
043 \\
044\end{array}$ & $\begin{array}{l}\text { layer } \\
\text { layer } \\
\text { layer } \\
\text { layer } \\
\text { layer }\end{array}$ & & & & & \\
\hline $\begin{array}{l}30-1,56-61 \\
30-1,98-104 \\
30-1 \\
30-1\end{array}$ & $\begin{array}{l}5 \\
6\end{array}$ & & $\begin{array}{l}045 \\
046\end{array}$ & $\begin{array}{l}\text { layer } \\
\text { layer }\end{array}$ & & & & & \\
\hline $30-3,80-86$ & 6 & & 047 & layer & & & & & \\
\hline $\begin{array}{l}31-1,33-40 \\
31-5,11-12 \\
33-1\end{array}$ & 7 & & 048 & $\begin{array}{l}\text { layer } \\
\text { layer }\end{array}$ & & & & & \\
\hline $\begin{array}{l}33-2,73-80 \\
33-2\end{array}$ & 7 & 77 & 050 & layer & & & & & \\
\hline $\begin{array}{l}33-5 \\
33-6,112-120 \\
34-3,19-25\end{array}$ & $\begin{array}{l}8 \\
6\end{array}$ & 113 & 051 & layer, graded & & & & & \\
\hline $\begin{array}{l}34-5,30-37 \\
35-3,21-30\end{array}$ & $\begin{array}{l}7 \\
9\end{array}$ & 28 & $\begin{array}{l}052 \\
053\end{array}$ & $\begin{array}{l}\text { layer } \\
\text { layer }\end{array}$ & & upper Miocene & & & \\
\hline $\begin{array}{l}36-4,112-122 \\
36-4 \\
36-4 \\
36-5 \\
36-6,28-32\end{array}$ & 10 & 118 & 054 & layer & & middle Miocene & & & \\
\hline $\begin{array}{l}37-2 \\
37-2 \\
37-2 \\
37-3 \\
38-2,26-31\end{array}$ & 5 & & 056 & layer & & & & & \\
\hline $\begin{array}{l}38-2 \\
38-4,16-20 \\
38-7\end{array}$ & 4 & & 057 & layer & & & & & \\
\hline
\end{tabular}


DSDP cores. In the present study, we define distinct ash layers as traceable ones and number them from top to bottom. Cadet and Fujioka (this volume) obtained a frequency curve of the volcanicity with geologic time by using the visual core description. They considered all ash layers and small pods or pockets as a record of volcanism, although small pods or pockets seem not to correlate among sites.

The curve obtained by Cadet and Fujioka shows a large, broad peak in the Pliocene and a small peak at the boundary between the lower and the middle Miocene at Sites 438 and 439 , whereas two definite separated peaks are observed in the upper and the lower Pliocene at Site 440 , at the trench slope bench. Although the curve at Site 440 has a double peak, the two curves at 438 and 439 and at 440 are quite similar. The double peak at Site 440 may be caused by sedimentation rate, bottom topography, current, degree of bioturbation, and many factors other than volcanic activity itself.

At Site 436, volcanic materials increase upward from Miocene to Recent. Furuta and Arai (this volume) counted the numbers of the volcanic ash layers and ashy pods or pockets and found that at Site 436 there were 56 volcanic ash layers and pods in the Pleistocene, 31 in the late Pliocene, 27 in the early Pliocene, 20 in the late Miocene, and 12 in the middle Miocene. The estimated frequency curve is different from that of the upper slope sites. The difference in number of ash layers on the oceanic plate is due to its continuous movement toward the Japanese Islands, which enables the volcanic glass gradually to reach Site 436 . Meanwhile, Sites 438,439 , and 440 are fixed relative to the Japanese Islands. It is very interesting to compare the two environments, oceanic plate and continental. Precise study of Leg 56 volcanic ash is necessary.

\section{Smear Slide Observation}

Smear slide observation carried out onboard shows a continuous downhole variation in volcanic glass content dispersed in sediment and sedimentary rock. In Unit 1 of Sites 438 and 439 , vitric volcanic glass composes up to 10 per cent of the sediment, then gradually decreases downward. At the boundary between the upper and middle Miocene, this component seems to disappear; however, in the lower Miocene it again increases gradually, often exceeding 10 per cent. In the lower Miocene, volcanic glass dispersed in sediment reaches a maximum content and rarely exceeds 20 per cent of the constituents in Lithologic Unit 3.

Lithologic Unit 3 (von Huene et al., 1978) consists of sandy claystone and diatomaceous claystone throughout, showing deep bathyal sedimentary environment. Burrow mottling, microfaults, and fractures are common. The vitric component of sediments has a plateau of approximately 20 per cent throughout Units 3 and 4, then gradually decreases downward and disappears in Units 5, 6, and 7. The frequency pattern in dispersed volcanic glass correlates well to that in volcanic ash layers and small pods or pockets. However, at Site 440, volcanic glass dispersed in sediments is not so variable throughout Holes 440, 440A, and 440B. At this site, vol- canic glass content always reaches 5 per cent or more, with slight local fluctuations. The frequency pattern of dispersed volcanic ash which distinguishes between the two sites is due to the sedimentation rate, topography, current, bioturbation, and many other factors in the depositional environment.

Smear slide observation shows volcanic glass to be predominately rhyolitic, with an almost transparent white appearance-quite different from the brown basaltic glass with a high refractive index which occurs sporadically throughout the sedimentary section. Nor is this rhyolitic glass representative of the predominant rhyolitic volcanism in the source region. This aspect will be discussed in the following section.

\section{Petrography of Volcanic Glass}

Table 2 presents a description of volcanic ash-type, color, and grain size-and shows the refractive index of volcanic glass and its constituent minerals. Experimental procedures are as follows: First, tephra samples are cleaned by an ultrasonic cleaner. Clay minerals and other dusts smaller than $5 \mu \mathrm{m}$ are sieved off with water. Next constituent minerals, type, color, and grain size of volcanic glass shard are examined under the microscope. Refractive indices are measured by the phasecontrast technique, using monochromatic illumination at a controlled constant room temperature of $24 \pm 0.2^{\circ} \mathrm{C}$. The margin of error of the indices of immersion liquid is less than \pm 0.0002 . The measured results are summarized by the maximum and minimum values of refractive indices in the tephra sample, the mean range in which more than 80 per cent of the measured refractive indices are concentrated, and the most frequent values of the refractive indices. Volcanic glass is usually separated into two types, pumice and bubble wall. Usually both types occur in the same ash layer. Figure 1 shows electron microscope photographs of volcanic glass samples in which large, tabular pumice predominates. These glasses were impregnated with an epoxy resin and prior to chemical analysis with an electron-probe microanalyzer. The maximum grain size of volcanic glass varies from $2.5 \mathrm{~mm}$ to $0.04 \mathrm{~mm}$, from very coarse sand to siltsize. The very coarse grained glass probably derives from a nearby source, whereas fine-grained glass probably has a more distant source. The settling velocity of volcanic glass was investigated by Fisher (1965), who demonstrated that bubble wall glass has a slightly higher rate of settling velocity than pumice, given a similar grain size.

Refractive indices of volcanic glass range from 1.496 to 1.529 , corresponding to a rhyolitic to rhyodacitic composition. A bimodal refractive index is observed in one ash layer.

Recently, Yoshikawa (1978) investigated the volcanic glass in the Osaka Group (Pliocene) and discussed the relative between refractive indices and the $\mathrm{SiO}_{2}$ and $\mathrm{Fe}_{2} \mathrm{O}_{3}$ content of volcanic glass. In acidic volcanic glass, the relationship is not clear. Data from Leg 57 shows no clear relationship either. Examination of the relationship between refractive index and chemical composition is necessary. 
TABLE 2

Description and Refractive Index of Volcanic Glass

\begin{tabular}{|c|c|c|c|c|c|c|c|c|c|}
\hline 1 & 2 & 3 & 4 & 5 & 6 & 7 & 8 & 9 & 10 \\
\hline $438-3-7,96-97$ & 1.0 & ++ & $\mathrm{Pl}++, \mathrm{Ho} \mathrm{Hy} \mathrm{Au} \mathrm{Mt}$ & $\mathrm{P}$ & $\mathrm{ClWh}$ & $1.503-1.520$ & $1.502-1.505$ & & 76.45 \\
\hline $438-10-3,124-125$ & 1.8 & +++ & $\mathrm{Pl}+++$, Hy Au Mt & Spg Fib & Wh Cl & $1.504-1.506$ & & $1.503+$ & 77.40 \\
\hline $438-10-5,80-81$ & 0.6 & ++ & $\mathrm{Pl+++}$, Ho Hy Au Mt & Spg Fib & inc rich & $1.503-1.505$ & & $1.505+$ & 76.35 \\
\hline $438-11-4,85-86$ & 0.1 & + & $\mathrm{Pl}++(\mathrm{Px}+)$ & P B & $\mathrm{ClWh}$ & $1.506-1.508$ & & $1.504+$ & 78.21 \\
\hline $438 \mathrm{~A}-2-5,20-21$ & $0.5-0.6$ & ++ & $\mathrm{Pl++}, \mathrm{Hy} \mathrm{Au} \mathrm{Mt}$ & $\mathbf{P}$ & $\mathrm{Cl}$ & $1.502-1.503$ & & & 78.41 \\
\hline $438 \mathrm{~A}-3-5,94-96$ & 1.5 & ++ & $\mathrm{Pl}++, \mathrm{Ho} \mathrm{Hy} \mathrm{Au}$ & Fib Str & Inc rich & $1.504-1.506$ & & $1.505+$ & \\
\hline $438 \mathrm{~A}-3-6,101-102$ & 0.5 & ++ & $\mathrm{Pl}++, \mathrm{Bi} \mathrm{Hy} \mathrm{Au} \mathrm{Mt}$ & $\mathrm{P}$ & $\mathrm{Cl}$ & $1.496-1.498$ & & $1.497+$ & 75.48 \\
\hline $438 \mathrm{~A}-6-4,22-23$ & $0.6-0.7$ & t+ & $\mathrm{Pl}+++\mathrm{Ho}(\mathrm{Cum}) \mathrm{Bi} \mathrm{Mt}$ & $\mathbf{P}$ & $\mathrm{Cl}$ & $1.498-1.499$ & & & \\
\hline $438 \mathrm{~A}-14-4,43-44$ & 1.2 & t+t & $\mathrm{Pl}++, \mathrm{Hy} \mathrm{Au} \mathrm{Mt}$ & Fib Spg & $\mathrm{Cl}$ & $1.506-1.509$ & & $1.508+$ & \\
\hline $438 \mathrm{~A}-16-3,143-145$ & 0.5 & & $\mathrm{Mt}+$ & P B & $\mathrm{Cl}$ & $1.501-1.503$ & & $1.502+$ & 76.30 \\
\hline $438 \mathrm{~A}-17-3,52-54$ & 1.0 & + & $\mathrm{Pl}++, \mathrm{Hy} \mathrm{Au} \mathrm{Mt} \mathrm{Ho}$ & $P$ & Wh & $1.508-1.511$ & & $1.510+$ & \\
\hline $438 \mathrm{~A}-34-1,53-54$ & 1.5 & & lith.++ & Fib Spg & Wh & $1.501-1.505$ & & & 76.45 \\
\hline $438 \mathrm{~A}-45-4,8-9$ & & +++ & $\mathrm{Pl}, \mathrm{Ho} \mathrm{Mt}$ & & & & & & \\
\hline $438 \mathrm{~A}-50-5,115$ & 2.5 & + & $\mathrm{Pl}+, \mathrm{Mf}-$ & Spg Fib & Wh & $1.504-1.506$ & & $1.505^{+}$ & \\
\hline $438 \mathrm{~B}-3-5,94-96$ & 1.0 & ++ & $\mathrm{Pl++}, \mathrm{Hy} \mathrm{Ho} \mathrm{Mt} \mathrm{Au}$ & $P$ & Wh & & & $(1.506+)$ & \\
\hline $\begin{array}{l}\text { 438B-9-3, 5-20 } \\
439-9-3,105-108\end{array}$ & 0.04 & + & $\begin{array}{l}\mathrm{Pl}++, \text { Cum Bi Mt } \\
\mathrm{Pl}+\text { (fine) }\end{array}$ & & $\mathrm{Cl}$ & $1.501-1.502$ & & $1.5015+$ & \\
\hline
\end{tabular}

Note: 1 = sample number, interval in $\mathrm{cm} ; 2=$ maximum grain size $; 3=$ amount of free crystal; $4=$ mineral composition; $5=$ type of volcanic glass; $6=$ color of volcanic glass; $7=$ range of refractive index; $8=$ mean range of refractive index; $9=$ mode of refractive index; $10=\mathrm{SiO}_{2}$ content of volcanic glass (in $\%$, measured with electron-probe microanalyzer). Abbreviations: $+=$ poor, $++=$ moderate,$++=$ abundant, $\mathrm{Pl}=$ plagioclase, $\mathrm{Ho}=$ hornblende, $\mathrm{Hy}=$ hypersthene, $\mathrm{Mt}=$ magmetite, $\mathrm{Px}=$ pyroxene, $\mathrm{Bi}=$ biotite, $\mathrm{Au}=$ augite, lith $=$ lithic, $\mathrm{Mf}=$ mafic mineral, $\mathrm{Cum}=$ cummingtonite $, \mathrm{Fib}=\mathrm{fibrous}, \mathrm{Str}=$ striped, $\mathrm{Spg}=$ spongy, $\mathrm{P}=$ pumice type, $\mathrm{B}=$ bubble wall type, $\mathrm{Cl}=$ clear, $\mathrm{Wh}=$ white, inc $=$ inclusion.

\section{Tephra Correlation}

Several investigators have successfully correlated deep sea tephra layers with source volcanic rocks on land (Ninkovich, 1968; Ninkovich \& Shackleton, 1975; Keller et al., 1978). Although deep sea tephra is several hundred or more $\mathrm{km}$ from the source volcanic rocks, it is correlatable using diagnostic features of volcanic ash. Bowles et al. (1973) succeeded in correlating ash layers with the reflected horizons of a $3.5-\mathrm{kHz}$ echogram off the coasts of Guatemala and Costa Rica. Leg 57 sites are located at about 200 to $300 \mathrm{~km}$ east of the Quaternary volcanic front on land, and frequent occurrence of many ash layers and ashy pods or pockets in Leg 57 sites produces fruitful correlations. Although volcanic glass at these sites shows very similar features downward throughout the cores, the drilling disturbance, post depositional tectonic disturbance, and bioturbation present serious problems. It was possible to correlate only two layers between Holes 438 and $438 \mathrm{~A}$, several hundred meters apart. From the point of view of biostratigraphy sedimentation rate, Samples 438-7-2, 48$50 \mathrm{~cm}$ and $438-7-2,103-105 \mathrm{~cm}$ may be reliably correlated with Sample 438A-5-3, 77-85 cm and with the ashy pods beneath this layer. Samples 438-3-6, 91-98 cm and 438A-2-2, 122-129 cm are also correlatable. Furuta and Arai (this volume) correlated the two uppermost ash layers in Hole 436 with the uppermost ones obtained during Hakuho-maru cruise $\mathrm{KH} 77-1$. In the area of
Sites 438, 439, 440, and 441 the uppermost sediments consist of such coarse-grained sand and pebbles that it was very difficult to obtain perfect cores. Two ash layers may be correlated with each other by chemical composition of glass and biostratigraphical age: Samples 438-11-5, 26-32 $\mathrm{cm}$ and 438A-6-4, 17-24 cm.

\section{MINERALOGY}

Table 2 shows the constituent minerals, as well as other features, of volcanic glass in ash layers. The constituent minerals observed are plagioclase, hornblende, hypersthene, augite, biotite, and magnetite. Cummingtonite occurs only rarely. Plagioclase is the most abundant, which is typical of rhyolitic volcanic rocks. Other constituent minerals vary from layer to layer. Chemical analyses were carried out with an electron-probe microanalyzer (see Tables 3-6). Table 3 shows the chemical composition of plagioclase in selected layers. It ranges from $\mathrm{An}_{73.43}$ in Sample 438A-24-1 to $\mathrm{An}_{7.57}$ in 438A-3-6, 101-102 cm. Volcanic glass contains an average of 70 per cent $\mathrm{SiO}_{2}$. High calcium plagioclase coexists with very acidic volcanic glass. From their study of the Tarumae volcano, Katsui et al. (1978) demonstrated that the bulk composition was intermediate whereas the liquid was very acidic and had erupted under conditions of very high vapor pressure.

Table 4 shows the chemical composition of pyroxene to be Wo-En-Fs. Clinopyroxene is augite and orthopy- 

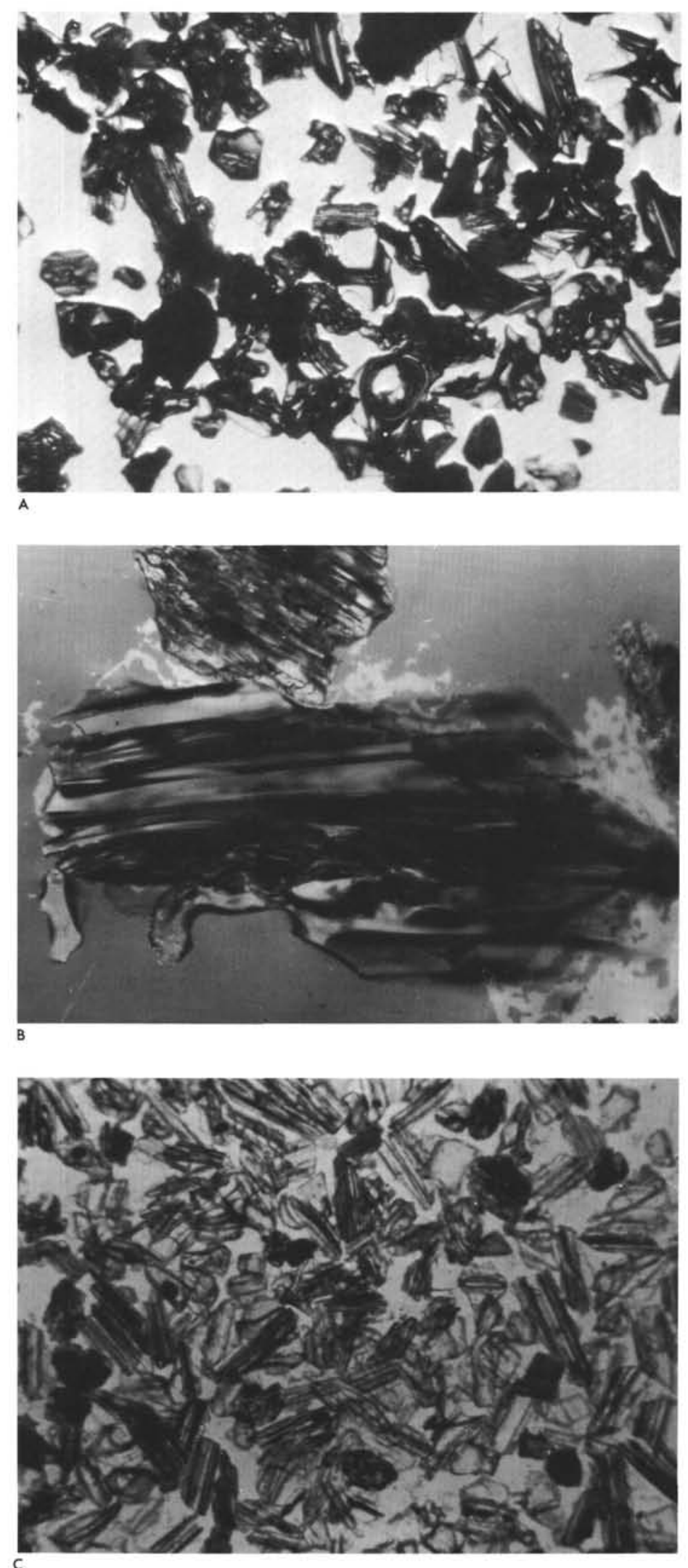

Figure 1. Microscopic photographs of volcanic glass. A. Sample 438A-6-3, 125-126 cm; B. Sample 438A-2-5, 109-110 $\mathrm{cm}$; C. Sample 438A-6-3, 125-126 cm, typical pumice-type glass with small vesicles. 
TABLE 3

Chemical Composition of Plagioclase (\%)

\begin{tabular}{|c|c|c|c|c|c|c|c|c|c|c|c|}
\hline Sample & $\begin{array}{c}438-7-7 \\
3 \mathrm{~cm}\end{array}$ & $\begin{array}{c}438-10-3 \\
124 \mathrm{~cm}\end{array}$ & $\begin{array}{c}438-11-1, \\
41 \mathrm{~cm}\end{array}$ & $\begin{array}{c}438-11-5 \\
30 \mathrm{~cm}\end{array}$ & \multicolumn{2}{|c|}{$438 \mathrm{~A}-2-2$} & $\begin{array}{c}438 \mathrm{~A}-3-6 \\
101 \mathrm{~cm}\end{array}$ & \multicolumn{3}{|c|}{$\begin{array}{c}438 \mathrm{~A}-6-2 \\
72 \mathrm{~cm}\end{array}$} & $\begin{array}{c}438 \mathrm{~A}-19-3 \\
95 \mathrm{~cm}\end{array}$ \\
\hline & & Core & & & Core & Core & & Core & Rim & & \\
\hline An & 40.98 & 62.59 & 44.63 & 69.11 & 45.97 & 49.30 & 7.57 & 52.43 & 47.06 & 43.24 & 40.09 \\
\hline $\mathrm{Ab}$ & 58.26 & 36.80 & 54.21 & 27.52 & 53.37 & 50.20 & 38.29 & 46.46 & 51.93 & 53.31 & 58.46 \\
\hline Or & 0.76 & 0.61 & 1.17 & 3.37 & 0.66 & 0.50 & 54.14 & 1.11 & 1.02 & 1.45 & 1.44 \\
\hline
\end{tabular}

roxene is hypersthene and ferrohypersthene. The range of chemical composition in zoned pyroxenes is very narrow.

Hornblende and biotite were also analyzed (Tables 5 and 6). The hornblendes and biotites are chemically homogeneous.

Fisher (1965) investigated the settling velocity of volcanic ash. He demonstrated that glass shards of different types settle at a velocity considerably lower than that of quartz grains. Based on their benthonic foraminiferal assemblages (von Huene et al., 1978), an outer shelf environment was inferred for the sedimentary section in the lower Miocene in Units 5 and 6; a shallow bathyal depth was inferred for Unit 4; and a deep bathyal environment was inferred throughout Units 2 and 3. Therefore it is possible that some volcanic shards are not deposited together with crystals of the same magma.

\section{CHEMICAL COMPOSITION OF VOLCANIC GLASS}

In order to estimate the nature of volcanic activity in the source region of each tephra layer, chemical analyses were carried out with a Model JXA-5 electron-probe microanalyzer, following the experimental procedures of Nakamura and Kushiro (1970). Volcanic ash samples are softly crushed and then cleaned with an ultrasonic cleaner. Glass is separated from its constituent minerals with a heavy liquid. Both glass and constituent minerals are respectively impregnated with an epoxy resin, then ground and polished with diamond paste for about three hours.

These conditions proved severe for labile glass. Because the alkali element count decreased with exposure to the electron beam, the beam was defocused. Variation in the experimental conditions gives slightly different values for the chemical composition of the glass. Thus total amount of oxide components ranges from about 85 per cent to 100 per cent. We were unable to pinpoint the amount of volatile material in each volcanic glass grain. A conventional examination was made of volcanic ash. Heating at $110^{\circ} \mathrm{C}$ and at $1050^{\circ} \mathrm{C}$ gives a rough idea of ignition loss. The preliminary result revealed a total ignition loss of 5.92 per cent for Sample $438 \mathrm{~A}-16-3,143-145 \mathrm{~cm}$. To estimate the volume more precisely, we consulted the data of other investigators. Yoshikawa (1978), who reported the chemical composition of volcanic glass in the Osaka Group, found that the $\mathrm{H}_{2} \mathrm{O}$ content in 24 upper Pliocene volcanic glasses ranges from 3.78 per cent to 5.59 per cent by weight. Because the similarity of chemical composition of the vol- canic glass between the Osaka Group and the Japan Trench area, it may be correct to regard the $\mathrm{H}_{2} \mathrm{O}$ content of rhyolitic glass as about 5 weight per cent. The chemical composition of obsidian and pitchstone is also very similar to that of rhyolitic volcanic glass (Kawano, 1950). Pitchstone always has a slightly higher $\mathrm{H}_{2} \mathrm{O}$ content than obsidian. Yoshikawa's result seems to accord well with pitchstone with regard to $\mathrm{H}_{2} \mathrm{O}$ content. In our study the low total oxide content of the glass may be due to hydration and/or to the experimental conditions. The hydration of volcanic glass is an important factor presently under investigation.

\section{General Remarks on the Chemical Composition of Volcanic Glass}

About 350 chemical analyses of volcanic glass were carried out with an electron-probe microanalyzer. Smith and Westgate (1969) and Westgate and Fulton (1975) performed such analyses, but many problems about the experimental conditions are still unsolved. Chemical composition of selected volcanic glass is listed in Table 7. $\mathrm{SiO}_{2}$ content ranges from 64 per cent to 80 per cent corresponding to that of dacite to rhyolite. These values are very similar to those of obsidian and pitchstone (Kawano, 1950). Samples often exceed 75 per cent $\mathrm{SiO}_{2}$, but this value seems slightly high compared with the other volcanic rocks. The $\mathrm{Al}_{2} \mathrm{O}_{3}$ content varies from about 10 per cent to 15 per cent, the average being 11 per cent. This value is similar to the volcanic rocks with the same $\mathrm{SiO}_{2}$ content. $\mathrm{TiO}_{2}$ content is a good means of distinguishing one ash layer from the other (Czamanske and Porter, 1965; Yokoyama, 1972). $\mathrm{TiO}_{2}$ content varies from 0.03 per cent to 0.65 per cent among the ash layers. $\mathrm{Fe}_{2} \mathrm{O}_{3}$ (total iron) and $\mathrm{MgO}$ vary considerably in different samples. The $\mathrm{FeO} * / \mathrm{MgO}$ ratio is also quite variable. In some samples, this ratio exceeds 100 . $\mathrm{MnO}$ is a minor constituent but distinct nevertheless layer by layer. $\mathrm{CaO}, \mathrm{Na}_{2} \mathrm{O}$, and $\mathrm{K}_{2} \mathrm{O}$ content changes a great deal both in the original magmatic process and in the hydration process of volcanic glass. During crystallization, the growth of plagioclase changes the $\mathrm{CaO}, \mathrm{Na}_{2} \mathrm{O}$, and $\mathrm{K}_{2} \mathrm{O}$ ratios of the magma. $\mathrm{K}_{2} \mathrm{O}$ content varies greatly, showing a maximum of about 5 per cent.

$$
\mathrm{SiO}_{2}-\left(\mathrm{Na}_{2} \mathrm{O}+\mathrm{K}_{2} \mathrm{O}\right)
$$

The chemical composition of the volcanic glass determined by electron-probe microanalysis is plotted in a $\mathrm{SiO}_{2}-\left(\mathrm{Na}_{2} \mathrm{O}+\mathrm{K}_{2} \mathrm{O}\right)$ diagram proposed by Kuno (1966) in order to discover if the glass belongs to a alkalic or nonalkalic rock series. In this diagram, only water-free 
TABLE 3 - Continued

\begin{tabular}{|c|c|c|c|c|c|c|c|c|c|c|c|}
\hline \multirow[t]{2}{*}{$438 \mathrm{~A}-24-1$} & \multicolumn{3}{|c|}{$\begin{array}{c}438 \mathrm{~A}-33-4 \\
73 \mathrm{~cm}\end{array}$} & & \multicolumn{3}{|c|}{$438 \mathrm{~A}-40-1$} & \multirow{2}{*}{$\begin{array}{l}\begin{array}{c}439-9-3 \\
105 \mathrm{~cm}\end{array} \\
\text { Core }\end{array}$} & \multicolumn{3}{|c|}{$\begin{array}{c}439-9-5 \\
49 \mathrm{~cm}\end{array}$} \\
\hline & & Core & Rim & & & & Core & & & & Rim \\
\hline 73.43 & 20.39 & 28.54 & 40.03 & 37.74 & 30.61 & 31.26 & 60.06 & 39.56 & 50.04 & 41.35 & 36.66 \\
\hline 26.35 & 77.53 & 69.38 & 58.53 & 60.70 & 68.07 & 66.74 & 39.13 & 59.01 & 49.06 & 56.39 & 60.50 \\
\hline 0.23 & 2.08 & 2.08 & 1.44 & 1.56 & 1.33 & 2.00 & 0.18 & 1.43 & 0.89 & 2.24 & 2.84 \\
\hline
\end{tabular}

TABLE 4

Chemical Composition of Pyroxene (\%)

\begin{tabular}{|c|c|c|c|c|c|c|c|c|c|c|c|c|c|c|}
\hline \multirow[t]{2}{*}{ Sample } & \multicolumn{2}{|c|}{$\begin{array}{c}438-10-3 \\
124 \mathrm{~cm}\end{array}$} & \multicolumn{3}{|c|}{$\begin{array}{c}438-11-1 \\
41 \mathrm{~cm}\end{array}$} & \multicolumn{2}{|c|}{$438 A-2-2$} & \multicolumn{6}{|c|}{$\begin{array}{c}438 \mathrm{~A}-6-2 \\
72 \mathrm{~cm}\end{array}$} & \multirow[t]{2}{*}{$\begin{array}{c}438 \mathrm{~A}-33-4, \\
73 \mathrm{~cm}\end{array}$} \\
\hline & $\mathrm{Cpx}$ & $\mathrm{Cpx}$ & $\mathrm{Cpx}$ & Opx & & Opx & & Opx & & & & Opx & Opx & \\
\hline & Core & Rim & Core & Core & Rim & Core & Rim & Core & & Core & Rim & Core & Rim & Opx \\
\hline Wo & 44.33 & 43.93 & 40.76 & 2.70 & 2.83 & 2.65 & 3.55 & 2.46 & 2.84 & 2.15 & 2.66 & 2.54 & 2.93 & 26.09 \\
\hline En & 40.36 & 39.99 & 39.17 & 58.70 & 58.45 & 65.15 & 65.08 & 45.56 & 46.80 & 47.07 & 45.63 & 45.72 & 44.81 & 44.21 \\
\hline Fs & 15.31 & 16.09 & 20.07 & 38.60 & 38.73 & 32.20 & 31.37 & 51.97 & 50.36 & 50.78 & 51.71 & 51.74 & 52.25 & 29.70 \\
\hline
\end{tabular}

Note: $\mathrm{Cpx}=$ clinopyroxene, $\mathrm{Opx}=$ orthopyroxene.

TABLE 5

\begin{tabular}{|c|c|c|}
\hline & Core & Rim \\
\hline 2 & 45.862 & 46.390 \\
\hline $\mathrm{O}_{2}$ & 0.528 & 0.486 \\
\hline${ }_{2} \mathrm{O}_{3}$ & 7.533 & 7.233 \\
\hline${ }_{2} \mathrm{O}_{3}$ & 13.658 & 13.285 \\
\hline & 0.245 & 0.345 \\
\hline $\mathrm{O}$ & 13.778 & 14.660 \\
\hline 0 & 11.473 & 11.821 \\
\hline${ }_{2} \mathrm{O}$ & 1.700 & 1.607 \\
\hline & 0.528 & 0.575 \\
\hline tal & 95.305 & 96.402 \\
\hline & 27.78 & 27.76 \\
\hline & 46.41 & 47.89 \\
\hline & 25.81 & 24.35 \\
\hline
\end{tabular}

data were plotted. Most of the data fall in low total alkali areas-that is, in the nonalkalic rock series. Some hydrated glass falls in the alkalic rock series, and the alteration or hydration of the glass is critical for its classification. Nevertheless, the trend of most volcanic glass is low alkalic-that is, nonalkalic rock series, whether calc-alkalic or tholeiitic (Figure 2).

$$
\mathrm{FeO}^{*}-\left(\mathrm{Na}_{2} \mathrm{O}+\mathrm{K}_{2} \mathrm{O}\right)-\mathrm{MgO}
$$

All the data are plotted in the FAM (iron-alkalimagnesium) diagram proposed by Wager and Deer (1939) (Figure 3). Although the data disperse in this diagram, most plots are along the F-A axis, which suggests that most of the volcanic glass belongs to the tholeiitic rock series represented by the Skaergaard trend. The most $\mathrm{FeO}^{*}$-(total iron as $\mathrm{FeO}$ ) enriched sample is plotted near the $\mathrm{FeO}^{*}$ corner of the Skaergaard trend. Generally, acidic volcanic glass is enriched in alkali compared with $\mathrm{FeO}^{*}$ and $\mathrm{MgO}$. In this diagram

TABLE 6

Chemical Composition of Biotite (\%)

\begin{tabular}{lccrcrc}
\hline & $\begin{array}{c}48 \mathrm{~A}-3-6, \\
101-102 \mathrm{~cm}\end{array}$ & $\begin{array}{c}\text { Recalculated } \\
\text { Composition }\end{array}$ & $\begin{array}{c}438 \mathrm{~A}-3-6, \\
101-102 \mathrm{~cm} \\
\text { Core }\end{array}$ & $\begin{array}{c}\text { Recalculated } \\
\text { Composition }\end{array}$ & \multicolumn{1}{c}{ Rim } & $\begin{array}{c}\text { Recalculated } \\
\text { Composition }{ }^{\text {a }}\end{array}$ \\
\hline $\mathrm{SiO}_{2}$ & 35.224 & 5.9686 & 33.301 & 5.8228 & 34.914 & 5.9547 \\
$\mathrm{TiO}_{2}$ & 3.421 & 0.4359 & 3.262 & 0.4289 & 3.429 & 0.4398 \\
$\mathrm{Al}_{2} \mathrm{O}_{3}$ & 15.642 & 3.1237 & 15.626 & 3.2201 & 15.425 & 3.1005 \\
$\mathrm{Fe}_{2} \mathrm{O}_{3} *$ & 17.329 & 2.4556 & 17.018 & 2.4885 & 17.184 & 2.4510 \\
$\mathrm{MnO}$ & 2.223 & 0.3190 & 2.222 & 0.3291 & 2.342 & 0.3384 \\
$\mathrm{MgO}$ & 10.557 & 2.6667 & 10.661 & 2.7790 & 10.747 & 2.7324 \\
$\mathrm{CaO}$ & 0.020 & 0.0037 & 0.041 & 0.0076 & 0.010 & 0.0019 \\
$\mathrm{Na}_{2} \mathrm{O}$ & 0.473 & 0.1556 & 0.426 & 0.1444 & 0.433 & 0.1431 \\
$\mathrm{~K}_{2} \mathrm{O}$ & 9.093 & 1.9656 & 8.877 & 1.9801 & 8.872 & 1.9301 \\
$\mathrm{Total}$ & 93.982 & 17.0943 & 91.435 & 17.2006 & 93.356 & 17.0919 \\
\hline
\end{tabular}

${ }^{\mathrm{a}}$ Number of ions of each element (not oxide) recalculated on the basis of 24 oxygen atoms. 
TABLE 7

Chemical Composition of Selected Volcanic Glass (\%)

\begin{tabular}{|c|c|c|c|c|c|c|c|c|c|c|c|c|}
\hline $\begin{array}{c}\text { Layer No. } \\
\text { Sample } \\
\text { (Interval in cm) }\end{array}$ & $\begin{array}{c}1 \\
438- \\
2-5, \\
109\end{array}$ & $\begin{array}{c}10 \\
438- \\
7-7 \\
3-4\end{array}$ & $\begin{array}{c}23 \\
438- \\
8-7 \\
11-12\end{array}$ & $\begin{array}{c}32 \\
438- \\
10-3, \\
124-125\end{array}$ & $\begin{array}{c}37 \\
438- \\
10-5, \\
80-81\end{array}$ & $\begin{array}{c}55 \\
438 \mathrm{~A}- \\
1-1, \\
70-72\end{array}$ & $\begin{array}{c}119 \\
438 \mathrm{~A}- \\
2-4 \\
132-133\end{array}$ & $\begin{array}{c}78 \\
438 \mathrm{~A}- \\
3-5 \\
94-96\end{array}$ & $\begin{array}{c}93 \\
438 \mathrm{~A}- \\
3-6 \\
101\end{array}$ & $\begin{array}{c}109 \\
438 \mathrm{~A}- \\
5-3 \\
81\end{array}$ & $\begin{array}{c}130 \\
438 \mathrm{~A}- \\
6-2 \\
72\end{array}$ & $\begin{array}{c}127 \\
438 \mathrm{~A}- \\
6-3, \\
125-126\end{array}$ \\
\hline $\mathrm{SiO}_{2}$ & 76.446 & 75.475 & 77.396 & 76.345 & 78.208 & 74.081 & 78.410 & 75.48 & 74.662 & 78.027 & 74.080 & 74.519 \\
\hline $\mathrm{TiO}_{2}$ & 0.218 & 0.184 & 0.137 & 0.294 & 0.241 & 0.429 & 0.210 & 0.19 & 0.173 & 0.232 & 0.148 & 0.049 \\
\hline $\mathrm{Al}_{2} \mathrm{O}_{3}$ & 11.136 & 11.366 & 11.403 & 10.407 & 11.343 & 12.910 & 11.391 & 11.38 & 11.830 & 11.155 & 11.496 & 11.633 \\
\hline $\mathrm{Fe}_{2} \mathrm{O}_{3} * \mathrm{a}$ & 2.055 & 1.472 & 1.479 & 1.316 & 1.366 & 3.078 & 1.188 & 3.97 & 1.377 & 1.151 & 1.256 & 1.711 \\
\hline $\mathrm{MnO}$ & 0.114 & 0.092 & 0.137 & 0.034 & 0.076 & 0.218 & 0.173 & 0.07 & 0.102 & 0.010 & 0.000 & 0.161 \\
\hline $\mathrm{MgO}$ & 0.238 & 0.197 & 0.154 & 0.217 & 0.192 & 1.774 & 0.125 & 0.32 & 0.190 & 0.173 & 0.110 & 0.058 \\
\hline $\mathrm{CaO}$ & 2.101 & 1.521 & 1.436 & 1.439 & 1.557 & 3.873 & 1.210 & 0.82 & 1.275 & 1.214 & 1.134 & 0.972 \\
\hline $\mathrm{Na}_{2} \mathrm{O}$ & 0.485 & 2.244 & 2.050 & 2.195 & 2.069 & 1.615 & 1.856 & 0.97 & 0.941 & 0.920 & 1.562 & 1.967 \\
\hline $\mathrm{K}_{2} \mathrm{O}$ & 0.886 & 1.417 & 1.852 & 2.905 & 2.101 & 0.963 & 2.292 & 1.08 & 1.658 & 1.803 & 2.728 & 3.008 \\
\hline Total & 93.679 & 93.968 & 96.044 & 95.151 & 97.153 & 98.942 & 96.854 & 94.28 & 92.208 & 94.684 & 92.515 & 94.076 \\
\hline $\mathrm{FeO} * * \mathrm{~b}$ & 1.850 & 1.325 & 1.331 & 1.184 & 1.229 & 2.770 & 1.069 & 3.573 & 1.239 & 1.036 & 1.130 & 1.540 \\
\hline $\mathrm{Al}_{2} \mathrm{O}_{3} / \mathrm{SiO}_{2}$ & 0.146 & 0.151 & 0.147 & 0.136 & 0.145 & 0.174 & 0.145 & 0.151 & 0.158 & 0.143 & 0.155 & 0.156 \\
\hline $\mathrm{FeO}^{* * \mathrm{~b}} / \mathrm{MgO}$ & 7.771 & 6.725 & 8.644 & 5.458 & 6.403 & 1.562 & 8.554 & 11.166 & 6.523 & 5.988 & 10.276 & 26.550 \\
\hline $\mathrm{CaO} / \mathrm{Na}_{2} \mathrm{O}$ & 4.332 & 0.678 & 0.700 & 0.656 & 0.753 & 2.398 & 0.652 & 0.845 & 1.355 & 0.132 & 0.726 & 0.494 \\
\hline $\mathrm{K}_{2} \mathrm{O} / \mathrm{Na}_{2} \mathrm{O}$ & 1.827 & 0.631 & 0.903 & 1.323 & 1.015 & 0.596 & 1.235 & 1.113 & 1.762 & 1.989 & 1.746 & 1.529 \\
\hline $\mathrm{Na}_{2} \mathrm{O}+\mathrm{K}_{2} \mathrm{O}$ & 39.64 & 70.63 & 72.43 & 78.45 & 74.58 & 36.20 & 77.65 & 34.49 & 64.52 & 69.25 & 77.58 & 75.69 \\
\hline $\mathrm{FeO}^{* * \mathrm{~b}}$ & 53.48 & 25.56 & 24.71 & 18.21 & 21.98 & 38.89 & 20.01 & 60.12 & 30.76 & 26.35 & 20.43 & 23.43 \\
\hline $\mathrm{MgO}$ & 6.88 & 3.80 & 2.86 & 3.34 & 3.43 & 24.91 & 2.34 & 5.38 & 4.72 & 4.40 & 1.99 & 0.88 \\
\hline $\mathrm{CaO}$ & 60.51 & 29.35 & 26.90 & 22.01 & 27.19 & 60.04 & 22.58 & 28.57 & 32.91 & 30.84 & 20.91 & 16.34 \\
\hline $\mathrm{Na}_{2} \mathrm{O}$ & 13.97 & 43.30 & 38.40 & 33.57 & 36.13 & 25.03 & 34.64 & 33.80 & 24.29 & 23.37 & 28.80 & 33.08 \\
\hline $\mathrm{K}_{2} \mathrm{O}$ & 25.52 & 27.34 & 34.69 & 44.43 & 36.69 & 14.93 & 42.78 & 37.63 & 42.80 & 45.80 & 50.29 & 50.58 \\
\hline $\mathrm{Na}_{2} \mathrm{O}+\mathrm{K}_{2} \mathrm{O}$ & 1.46 & 3.90 & 4.06 & 5.36 & 4.29 & 2.61 & 4.28 & 2.17 & 2.82 & 2.88 & 4.64 & 5.29 \\
\hline $\mathrm{SiO}_{2}$ & 81.60 & 80.32 & 80.58 & 80.24 & 80.50 & 74.87 & 80.96 & 80.06 & 80.97 & 82.41 & 80.07 & 79.21 \\
\hline
\end{tabular}

${ }^{\mathrm{a}} \mathrm{Fe}_{2} \mathrm{O}_{3}{ }^{*}=$ total iron calculated as $\mathrm{Fe}_{2} \mathrm{O}_{3}$.

$\mathrm{b} \mathrm{FeO}^{* *}=$ total iron calculated as $\mathrm{FeO}$.

one volcanic ash sample obtained in one layer is higher in $\mathrm{MgO}$ than other samples. This shows that it is composed of several volcanic glasses with different chemical compositions. Two possibilities suggest themselves: one is that several eruptions of different volcanoes in the source region may take place at almost the same time and that their chemical compositions are slightly different. The second possibility is that several eruptions of the same volcano may take place within a very short time span and the degree of magma differentiation may be slightly different. In the present case, the two possibilities are equally important.

In Figure 4, two different ash layers which may be correlated with each other are tested for three components: $\mathrm{FeO}^{*}, \mathrm{MgO}$, and total alkali. Therefore the correlation of each ash layer is more reliable than if the refractive index alone were used. At the same time, the three additional components, $\mathrm{CaO}, \mathrm{Na}_{2} \mathrm{O}$, and $\mathrm{K}_{2} \mathrm{O}$, are useful indicators for distinguishing the layers. Figure 4 shows the relationship between two different layers, Sample 438-11-5, 30-31 cm and Sample 438A-6-4, 22$23 \mathrm{~cm}$. In this figure, A shows the $\mathrm{FeO} *-\mathrm{MgO}-\left(\mathrm{Na}_{2} \mathrm{O}\right.$ $+\mathrm{K}_{2} \mathrm{O}$ ) relationship and $\mathrm{B}$ the $\mathrm{K}_{2} \mathrm{O}-\mathrm{CaO}-\mathrm{Na}_{2} \mathrm{O}$ relationship. It is clear from this diagram that the two samples have the same chemical composition. Biostratigraphic results support this evidence. Both ash layers belong to the upper Pliocene, and ratios FAM and $\mathrm{CaO}-$ $\mathrm{Na}_{2} \mathrm{O}-\mathrm{K}_{2}$ are concentrated in very narrow and overlapping areas. Thus it is valid to correlate the two layers with each other.
This method of correlation is more reliable than other methods and is quite useful for surrounding areas, such as the Northeast Japan Arc, which supply similar volcanic ash materials. In northern Honshu, the famous upper Pliocene Shirakawa dacite is widely distributed. The correlation of the two aforementioned tephra layers with the Shirakawa dacite of similar age remains for future investigation.

As stated earlier, the chemical composition of the volcanic glass is quite similar to that of obsidian and pitchstone. Fresh obsidian and pitchstone are plotted in the diagram in Figure 5. The data of all the volcanic glass show a close genetic similarity.

\section{DISCUSSION}

As stated in the previous sections, the frequency curve derived from the number of ash layers constitutes a record of the volcanicity near the Japan Trench sites. The land volcanism of the Tohoku region of Honshu has been summarized by many investigators (Sugimura et al., 1963; Kuno, 1966; Horikoshi, 1976). Figure 6 shows Horikoshi's estimation of volcanic activity in the Tohoku arc from approximately 30 m.y.B.P. to Recent. In the Quaternary, the volume of volcanic rocks is large near the volcanic front and is mainly tholeiitic in composition, becoming more alkalic as it moves west of the front. This relationship was well summarized by Kuno (1966) in many other circum-Pacific arcs. Moreover, the volume of volcanic activity in the Tertiary is four times that of the Quaternary. According to Horikoshi's es- 
TABLE 7 - Continued

\begin{tabular}{|c|c|c|c|c|c|c|c|c|c|c|c|c|c|}
\hline $\begin{array}{c}115 \\
438 \mathrm{~A}- \\
6-4 \\
22-23\end{array}$ & $\begin{array}{c}139 \\
438 \mathrm{~A}- \\
14-4, \\
43-44\end{array}$ & $\begin{array}{c}146 \\
438 \mathrm{~A}- \\
17-3 \\
52-54\end{array}$ & $\begin{array}{c}160 \\
438 \mathrm{~A}- \\
19-3 \text {, } \\
95-96\end{array}$ & $\begin{array}{c}164 \\
438 \mathrm{~A}- \\
24-1 \\
98\end{array}$ & $\begin{array}{c}172 \\
438 \mathrm{~A}- \\
24-4 \\
60\end{array}$ & $\begin{array}{c}179 \\
438 \mathrm{~A}- \\
24-6 \\
85\end{array}$ & $\begin{array}{c}185 \\
438 \mathrm{~A}- \\
27-3 \\
83-84\end{array}$ & $\begin{array}{c}199 \\
438 \mathrm{~A}- \\
33-6, \\
119-120\end{array}$ & $\begin{array}{c}211 \\
438 \mathrm{~A}- \\
34-1 \\
53-54\end{array}$ & $\begin{array}{c}221 \\
438 \mathrm{~A}- \\
40-1 \\
39\end{array}$ & $\begin{array}{c}233 \\
438 \mathrm{~A}- \\
50-5 \\
115\end{array}$ & $\begin{array}{c}243 \\
438 \mathrm{~B}- \\
9-3 \\
5-6\end{array}$ & $\begin{array}{l}253 \\
439- \\
7-1, \\
125\end{array}$ \\
\hline 75.879 & 76.300 & 74.15 & 75.914 & 72.470 & 75.94 & 77.83 & 73.023 & 74.33 & 76.447 & 73.022 & 74.046 & 75.39 & 74.221 \\
\hline 0.135 & 0.327 & 0.19 & 0.103 & 0.080 & 0.08 & 0.15 & 0.367 & 0.30 & 0.097 & 0.109 & 0.114 & 0.06 & 0.543 \\
\hline 11.514 & 11.208 & 12.12 & 13.299 & 11.482 & 11.52 & 11.59 & 13.434 & 11.67 & 11.412 & 11.688 & 12.287 & 11.90 & 15.231 \\
\hline 0.630 & 2.292 & 4.63 & 1.322 & 1.221 & 1.97 & 1.12 & 1.737 & 4.30 & 1.579 & 1.388 & 1.596 & 1.96 & 3.184 \\
\hline 0.103 & 0.073 & 0.08 & 0.079 & 0.072 & 0.07 & 0.04 & 0.095 & 0.11 & 0.075 & 0.034 & 0.205 & 0.01 & 0.213 \\
\hline 0.070 & 0.282 & 0.30 & 0.092 & 0.102 & 0.16 & 0.14 & 0.203 & 0.29 & 0.023 & 0.119 & 0.128 & 0.08 & 0.511 \\
\hline 0.547 & 1.942 & 1.13 & 1.232 & 1.000 & 2.12 & 1.03 & 3.555 & 1.15 & 1.025 & 1. & & 0.30 & 2.679 \\
\hline 2.852 & 1.090 & 1.87 & 1.130 & 2.395 & 0.50 & 1.03 & 3.397 & 0.80 & 2.100 & 2. & 34 & 0.79 & 0.290 \\
\hline 4.117 & 1.868 & 1.07 & 2.628 & 2.315 & 1.26 & 2.52 & 1.386 & 1.60 & 1.672 & 2.815 & 1.681 & 3.07 & 1.543 \\
\hline 95.847 & 95.382 & 95.54 & 95.798 & 91.135 & 93.59 & 95.44 & 97.196 & 94.56 & 94.432 & 92.924 & 94.227 & 93.57 & 98.415 \\
\hline 0.567 & 2.063 & 4.167 & 1.190 & 1.099 & 1.773 & 1.01 & 1.563 & 3.8 & 1.421 & 1.249 & 1.436 & 1.764 & 2.866 \\
\hline 0.152 & 0.147 & 0.163 & 0.175 & 0.158 & 0.152 & 0.149 & 0.184 & 0.157 & 0.149 & 0.160 & 0.166 & 0.158 & 0.205 \\
\hline 8.100 & 7.315 & 13.890 & 12.933 & 10.774 & 11.081 & 7.200 & 7.701 & 13.345 & 61.787 & 10.497 & 11.222 & 22.050 & 5.608 \\
\hline 0.192 & 1.782 & 0.604 & 1.090 & 0.418 & 4.240 & 1.000 & 1.047 & 1.438 & 0.488 & 0.563 & 0.646 & 0.380 & 9.238 \\
\hline 1.444 & 1.714 & 0.572 & 2.326 & 0.967 & 2.520 & 2.447 & 0.408 & 2.000 & 0.796 & 1.173 & 0.663 & 3.886 & 5.321 \\
\hline 91.63 & 55.78 & 39.69 & 74.56 & 79.68 & 47.6 & 75.56 & 73.0 & 36.59 & 72.3 & 79.2 & 72. & 67.6 & 35.18 \\
\hline 7.54 & 38.90 & 56.26 & 23.61 & 18. & 4 & 21.46 & 23. & 58 & 27. & 18.97 & 24.85 & 30.93 & 55.01 \\
\hline 0.92 & 5.32 & 4.05 & 1.83 & 1.73 & 4.33 & 2.98 & 3.10 & 4.42 & 0.44 & 1.81 & 2.21 & 1.40 & 9.81 \\
\hline 7.28 & 39.63 & 27.76 & 24.69 & 17.51 & 54.64 & 22.49 & 42.64 & 32.39 & 21.37 & 20.56 & 27.96 & 7.21 & 59.38 \\
\hline 37.95 & 22.24 & 45.95 & 22.65 & 41.94 & 12.89 & 22.49 & 40.74 & 22.54 & 43.78 & 36.56 & 43.31 & & 6.43 \\
\hline 54.78 & 38.12 & 26.29 & 52.67 & 40.54 & 32.47 & 55.02 & 16.62 & 45.07 & 34.86 & 42.88 & 28.73 & 73.80 & 34.20 \\
\hline 7.27 & 3.10 & 3.08 & 3.92 & 5.17 & 1.88 & 3.72 & & 2.54 & 3.99 & 5.61 & 4.47 & 4.13 & 1.86 \\
\hline 79.17 & 79.99 & 77.61 & 79.24 & 79.52 & 81.14 & 81.55 & 75.13 & 78.61 & 80.95 & 78.58 & 78.58 & 80.57 & 75.42 \\
\hline
\end{tabular}

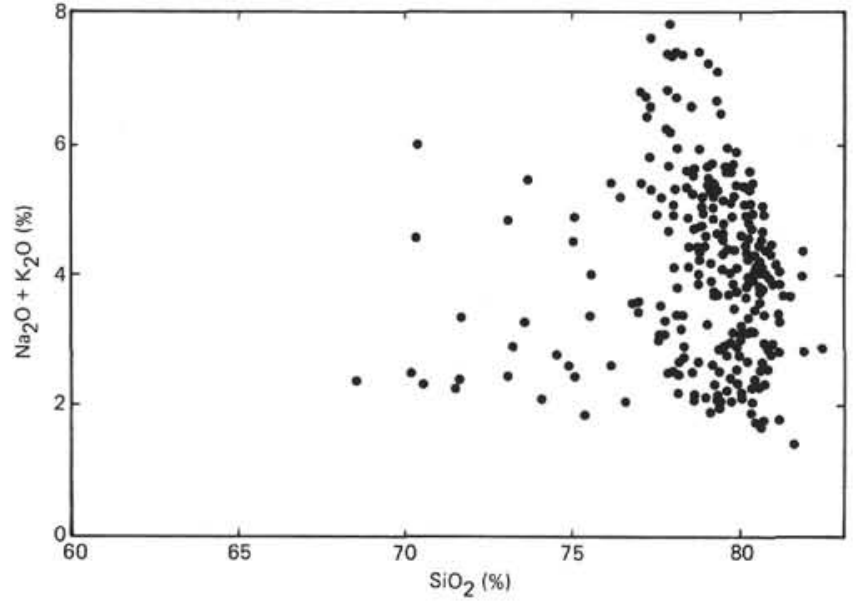

Figure 2. Alkali-silica diagram of volcanic glass from Leg 57. Components are recalculated water-free.

timate, the amount of volcanic rock in his "Initial Stage" (27 m.y.B.P.-13 m.y.B.P.) is greater than that of his "Stational Stage" (after 13 m.y.B.P.). Horikoshi's estimate and that of Cadet and Fujioka differ in that land volcanicity is very intense during the Miocene, whereas it is intense at the Japan Trench sites during the Pliocene. This discrepancy is due mainly to the fact that volcanic activity at DSDP sites reflects the more explosive acidic rhyolitic ash, so that the curve obtained by Cadet and Fujioka shows only acidic volcanism and contains no basic volcanic products.

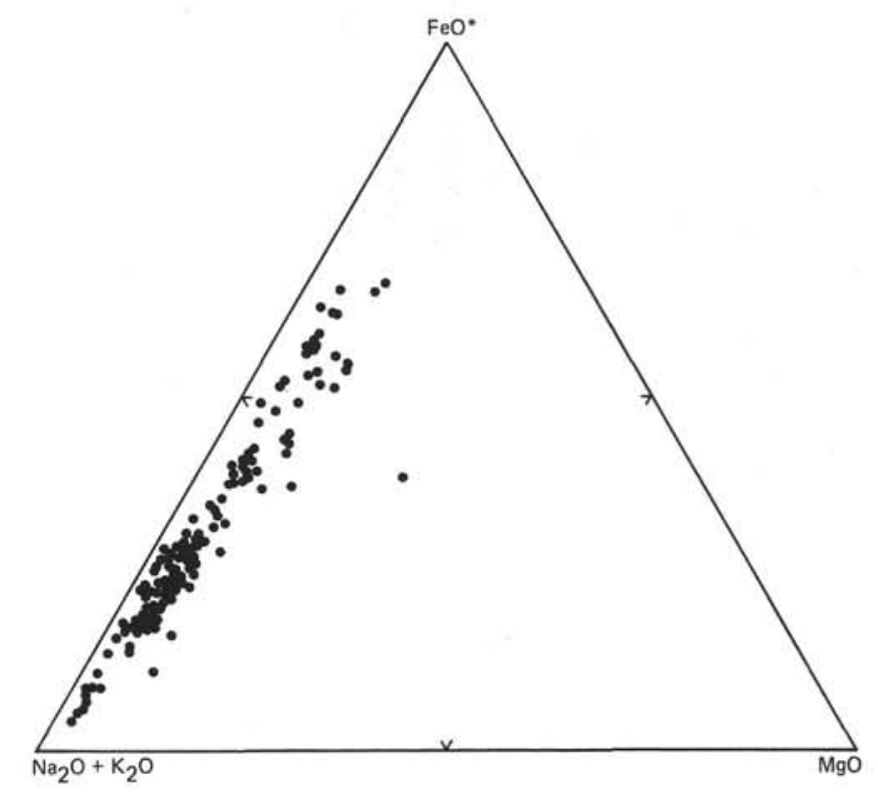

Figure 3. $\mathrm{FeO}^{*}-\left(\mathrm{Na}_{2} \mathrm{O}+\mathrm{K}_{2} \mathrm{O}\right)-\mathrm{MgO}$ diagram of volcanic glass. $\mathrm{FeO} *$ represents total iron calculated as $\mathrm{FeO}$.

The factors controlling the mode of eruption are very complicated (Verhoogen, 1955; Aramaki, 1975). One of these is the chemical composition of magma (Katsui et al., 1978). Generally, acidic magma contains more volatile components than basaltic magma. Rhyolitic volcanic glass seems to be more widely distributed than 

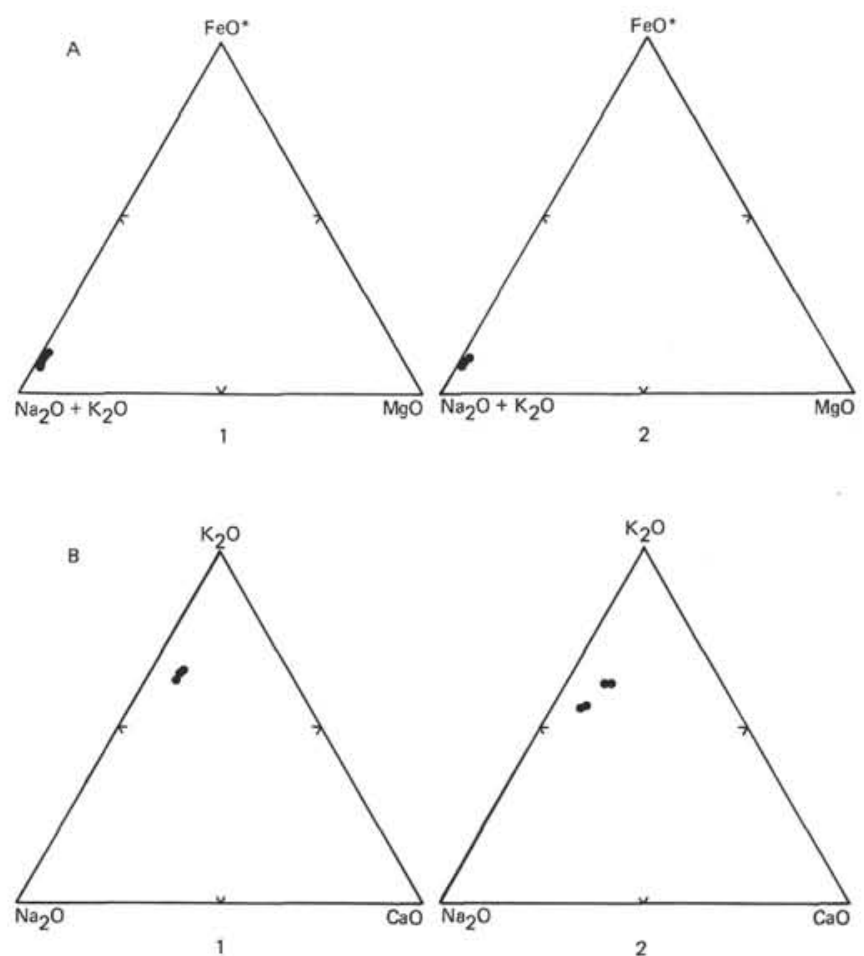

Figure 4. A triangle plot of correlation of two ash layers. 1. Sample 438-11-5, 30-31 cm; 2. Sample 438A $6-4,22-23 \mathrm{~cm}$; A. $\mathrm{FeO}^{*}-\left(\mathrm{Na}_{2} \mathrm{O}+\mathrm{K}_{2} \mathrm{O}\right)-\mathrm{MgO}$ diagram; B. $\mathrm{K}_{2} \mathrm{O}-\mathrm{Na}_{2} \mathrm{O}-\mathrm{CaO}$ diagram.

basaltic glass. For example, Ninkovich (1968) correlated deep sea ashes with those on land in the New Zealand Taup-Rotorua area. In his data on acidic and basic tephra, volcanic glass distribution depends mainly on its chemical composition. Acidic volcanic glass is often found more than $1000 \mathrm{~km}$ from the source (Machida and Arai, 1976).

As stated earlier, basaltic glass at DSDP sites is rare compared with rhyolitic glass. Further correlation be- tween on land and deep sea tephra in the Tohoku arc is necessary, and the frequency curve of volcanicity needs revision.

The chemical composition of volcanic glass discussed in Section III has been obtained from fairly fresh samples. However, most of the volcanic glass had suffered from hydration and/or alteration to various degrees. Some of the basaltic glass observed in smear slides had been altered to palagonite. Also, in some rhyolitic glass, very weak birefringence shows up under the microscope. Glass samples were examined by X-ray diffractometer, revealing a broad peak of clay minerals. Alteration of glass shards is the most severe problem in determining magma type. Chemical scanning patterns in Figure 7, $\mathrm{A}$ and $\mathrm{B}$ show the $\mathrm{Ca}-\mathrm{K} \alpha, \mathrm{Fe}-\mathrm{K} \alpha$, and $\mathrm{Mg}-\mathrm{K} \alpha$ relationships in a single grain of volcanic glass. Sample 438A-33-6, 119-120 $\mathrm{cm}$ has a homogeneous pattern throughout the grain, whereas 438B-9-3, 5-6 cm has a heterogeneous pattern. In the latter case, $\mathrm{Ca}, \mathrm{Mg}$, and $\mathrm{Fe}$ decrease near the periphery of the grain, forming a regular chemical gradient, and $\mathrm{Si}, \mathrm{Al}$, and $\mathrm{Fe}$ decrease uniformly near the vesicle. This may indicate secondary leaching of the cation toward the rim. These chemical scanning patterns are valid means of determining the hydration and/or alteration processes of the volcanic glass.

The $\mathrm{K}_{2} \mathrm{O}, \mathrm{Na}_{2} \mathrm{O}$, and $\mathrm{CaO}$ relationships of many volcanic glasses were examined, and several examples were shown in Figure 5. The $\mathrm{K}_{2} \mathrm{O} / \mathrm{Na}_{2} \mathrm{O}$ ratio of volcanic glass seems to increase toward the rim of the grain, but not in all cases. A similar trend was observed by Moore (1966) in the glass samples of altered pillow lavas. His results show clearly the increase of $\mathrm{K}_{2} \mathrm{O}$ content as hydration increases. The $\mathrm{K}_{2} \mathrm{O} / \mathrm{Na}_{2} \mathrm{O}$ ratio of each kind of volcanic glass is variable. The $\mathrm{K}_{2} \mathrm{O} / \mathrm{Na}_{2} \mathrm{O}$ ratio in sea water is very small compared with the same ratio in the glass. We can therefore assume that leaching of $\mathrm{K}_{2} \mathrm{O} / \mathrm{Na}_{2} \mathrm{O}$, if it does take place, depends not on fresh sea water but on interstitial water.

Recently, Hein et al. (1978) investigated the diagenesis of volcanic ash recovered during DSDP Leg 19 in the
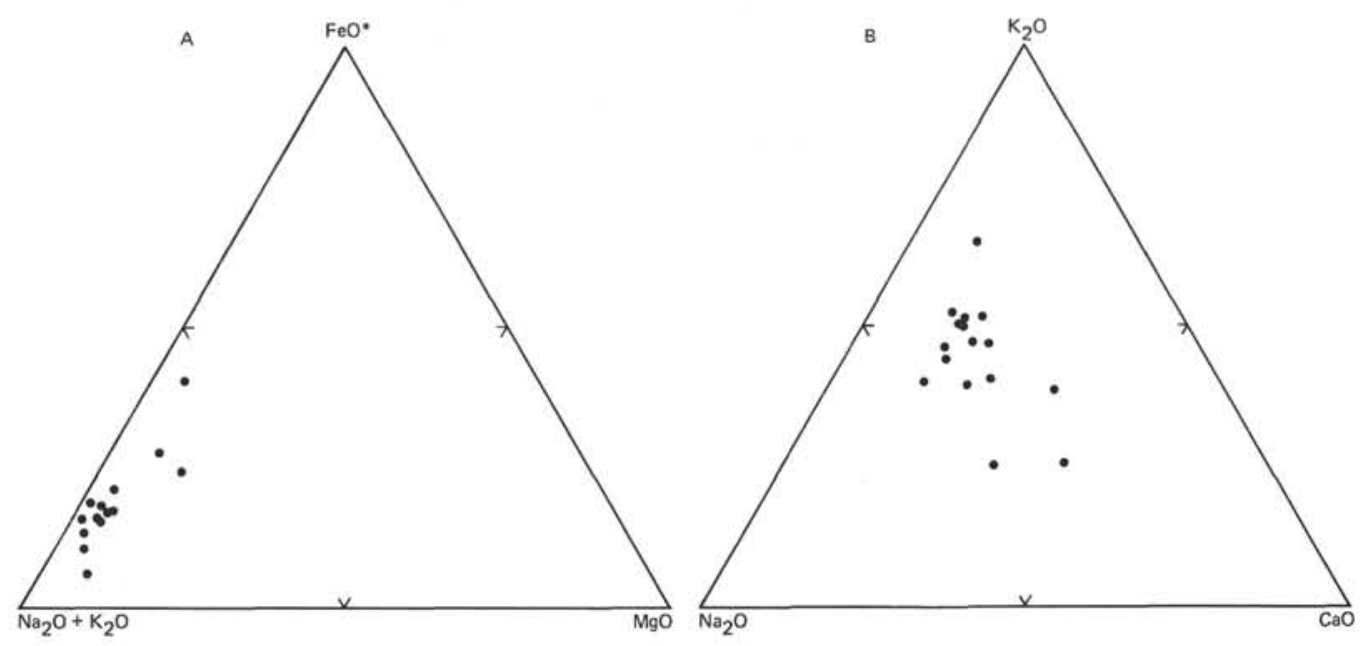

Figure 5. Ternary diagrams for Japan obsidian and pitchstone. (Data from Kawano, 1950; diagrams same as in Figure 4.) 


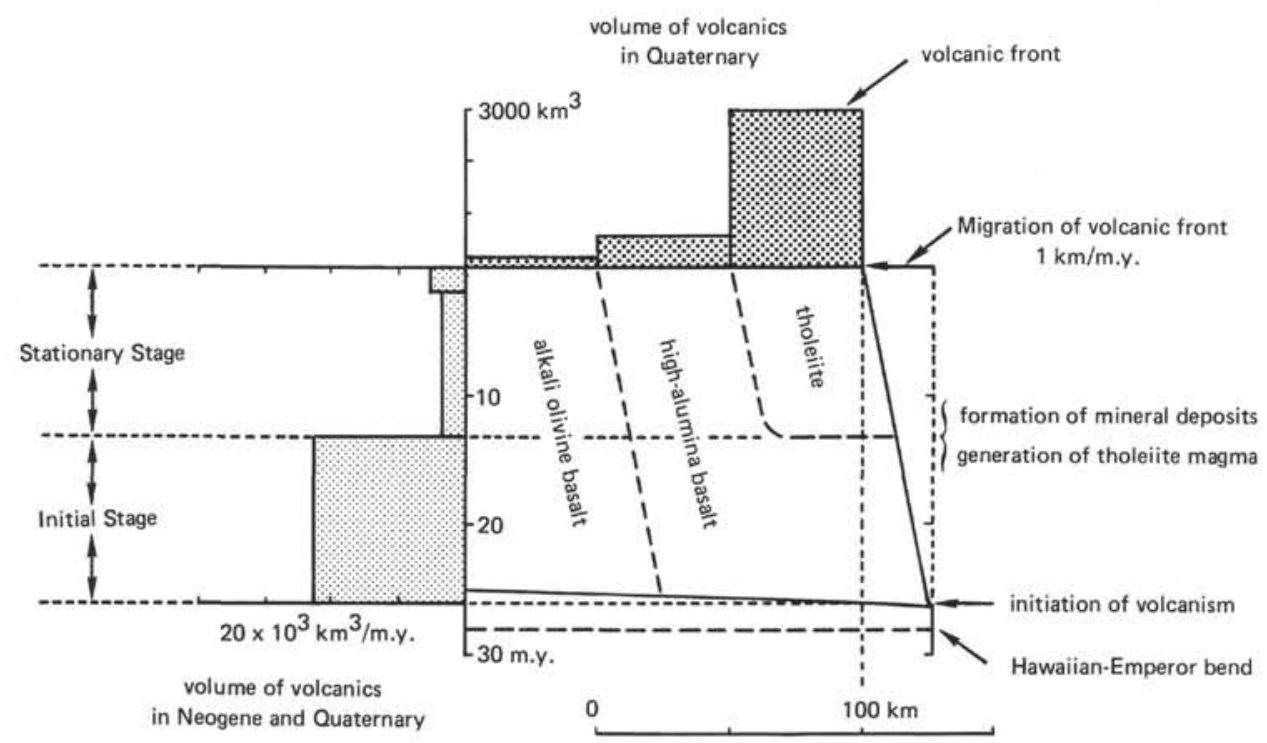

Figure 6. Modal volume of volcanic rocks in the Tohoku Arc, Japanese Islands.

(From Horikoshi, 1976.)

Bering Sea. Downhole temperature measurement at Leg 19 sites indicates $40^{\circ} \mathrm{C}$ or less at the bottom and an isotopic temperature, measured by oxygen, of $70^{\circ} \mathrm{C}$. However, at Site 439 , bottom temperature is less than $29^{\circ} \mathrm{C}$ and observed alteration seems very weak compared with that of Leg 19.

Settling velocity of volcanic glass and mafic minerals are critical to determining the magmatic conditions of eruption. Fisher (1965) investigated the settling velocity of various types of glass using Stoke's law. If his results are valid, settling velocities of mafic heavy minerals and glass of the same radii are considerably different. Whether or not mafic minerals coexist with groundmass glass is a critical question which requires an answer in the near future.

\section{SUMMARY}

1) Explosive acidic volcanism took place with the greatest intensity during the Pliocene, judging from the volcanic ash layers of Leg 57.

2) Three volcanic ash layers were correlated from the standpoint of biostratigraphy and chemical composition.

3) The chemical composition of the volcanic glass shows that in the $\mathrm{SiO}_{2}-\left(\mathrm{Na}_{2} \mathrm{O}+\mathrm{K}_{2} \mathrm{O}\right)$ diagram it belongs in the nonalkalic rock series and that in the $\mathrm{FeO}^{*}$ $\left(\mathrm{Na}_{2} \mathrm{O}+\mathrm{K}_{2} \mathrm{O}\right)-\mathrm{MgO}$ diagram it exhibits trends similar to those of a tholeiitic rock series.

4) The $\mathrm{K}_{2} \mathrm{O} / \mathrm{Na}_{2} \mathrm{O}$ ratio of the slightly hydrated volcanic glass increases toward the rim in a single grain of glass. This trend is very similar to the palagonitization reported by many investigators.

\section{ACKNOWLEDGMENTS}

The authors would like to express sincere thanks to Drs. Noriyuki Nasu, H. Kagami, K. Kobayashi, J. Segawa, H.
Tokuyama, and T. Ishii for their valuable suggestions and critical reading of this manuscript.

\section{REFERENCES}

Aramaki, S., 1975. Classification and mechanism of volcanic eruptions. Bull. Volcanol. Soc. Jpn. Ser. 2, 20, 205-221.

Bowles, F. A., Sack, R. N., and Carmichael, I. S. E., 1973. Investigation of deep-sea volcanic ash layers from equatorial Pacific cores. Geol. Soc. Am. Bull., 84, 2371-2388.

Czamanske, G. K., and Porter, S. C., 1965. Titanium dioxide in pyroclastic layers from volcanoes in the Cascade Range. Science, 150, 1022-1025.

Fisher, R. V., 1965. Settling velocity of glass shards. Deep Sea Res., 12, 345-353.

Hein, J. R., and Scholl, D. W., 1978. Diagenesis and distribution of late Cenozoic volcanic sediment in the southern Bering Sea. Geol. Soc. Am. Bull., 89, 197-210.

Hein, J. R., Scholl, D. W., and Miller, J., 1978. Episodes of Aleutian ridge explosive volcanism. Science, 199, 137-141.

Horikoshi, E., 1976. Development of late Cenozoic petrogenic provinces and metallogeny in Northeast Japan. Geol. Assoc. Can. Spec. Pap., 14, 121-142.

Katsui, Y., Oba, Y., and Saya, T., 1978. Records of volcanic eruptions in historic times and estimation of future eruptions. Bull. Volcanol. Soc. Jpn. Ser. 2, 23, 41-52.

Kawano, J., 1950. Natural glasses in Japan. Rep. Geol. Surv. Jpn., 137. (In Japanese with English abstract).

Keller, J., Ryan, W. B. F., Ninkovich, D., and Altherr, R., 1978. Explosive volcanic activity in the Mediterranean over the past 200,000 yrs as recorded in deep sea sediments. Geol. Soc. Am. Bull., 89, 591-604.

Kennett, J. P., and Thunell, R. C., 1975. Global increase in Quaternary explosive volcanism. Science, 187, 497-503.

Kuno, H., 1966. Laterial variation of basaltic magma type across continental margins and island arcs. Bull. Volcanol. Soc. Jpn. Ser. 2, 29, 195-222.

Machida, H., and Arai, F., 1976. The very widespread tephra - The Aira-Tn ash. Science, 46, 339-347. (In Japanese)

Moore, J. G., 1966. Rate of palagonitization of submarine basalt adjacent to Hawaii. U.S. Geol. Surv. Prof. Pap., 550-D, 163-171. 

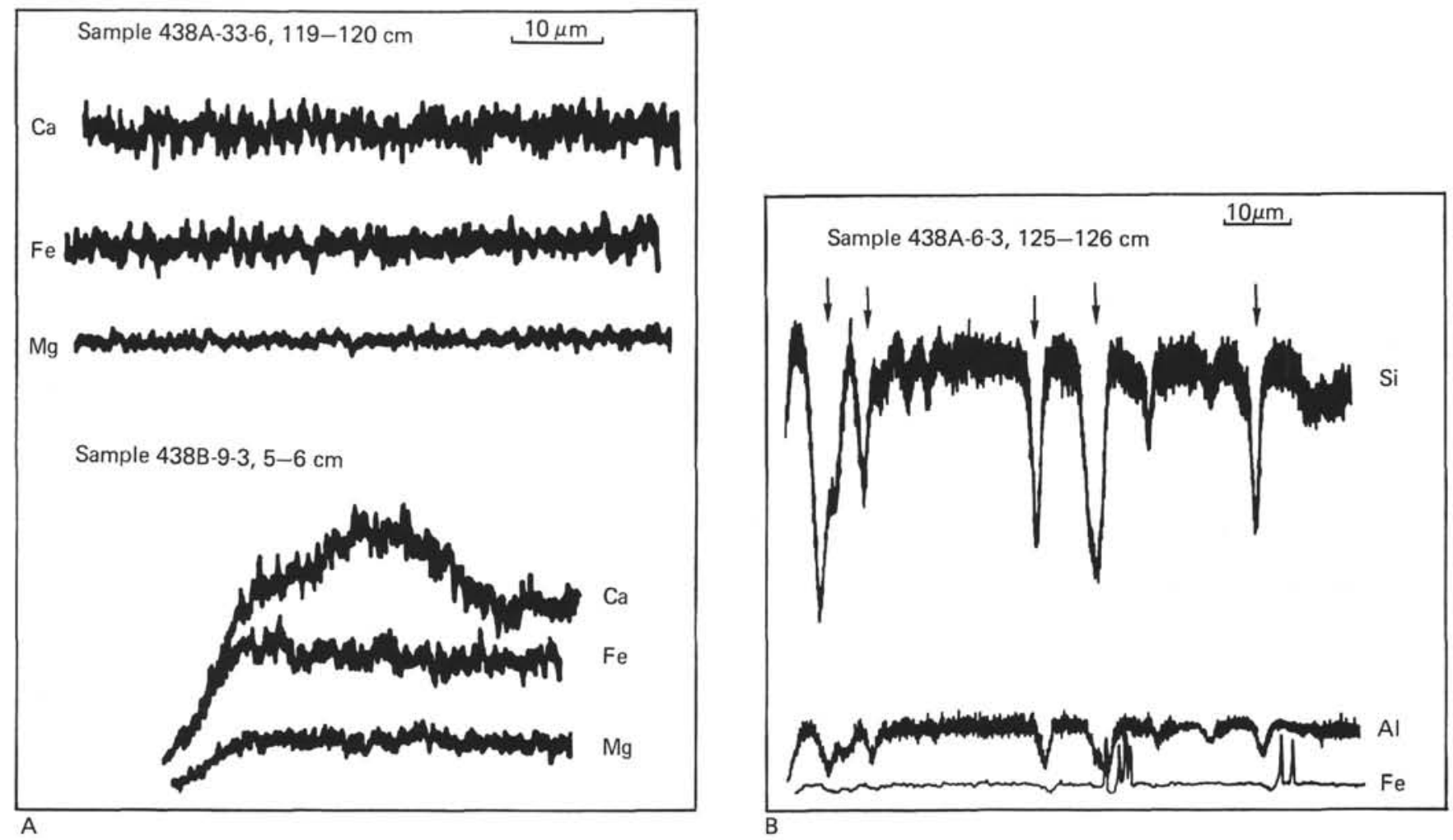

Figure 7. Chemical scanning pattern of volcanic glass shard. A. Ca-Mg-Fe relation. The upper sample shows a homogeneous pattern; the lower shows remarkable variation from core to rim within a single volcanic glass grain. B. $\mathrm{Si}-\mathrm{Al}-\mathrm{Fe}$ relation. Si and $\mathrm{Fe}$ decrease gradually toward the vesicles.

Nakamura, Y., and Kushiro, I., 1970. Compositional relations of coexisting orthopyroxene, pigeonite and augite in a tholeiitic andesite from Hakone Volcano. Contr. Mineral. Petrol., 26, 265-275.

Ninkovich, D., 1968. Pleistocene volcanic eruptions in New Zealand recorded in deep-sea sediments. Earth Planet. Sci. Lett., 4, 89-102.

Ninkovich, D., and Donn, W. L., 1976. Explosive Cenozoic volcanism and climatic implications. Science, 194, 899-906.

Ninkovich, D., and Shackleton, N. J., 1975. Distribution, stratigraphic position and age of ash layer " $L$ " in the Panama Basin region. Earth Planet. Sci. Lett., 27, 20-34.

Smith, D. G. W., and Westgate, J. A., 1969. Electron probe technique for characterizing pyroclastic deposits. Earth Planet. Sci. Lett., 5, 313-319.

Sugimara, A., Matsuda, T., Chinzei, K., and Nakamura, K., 1963. Quantitative distribution of late Cenozoic volcanic materials in Japan. Bull. Volcanol. Soc. Jpn., Ser. 2, 26, $125-140$.
Verhoogen, J., 1955. Mechanics of ash formation. Am. J. Sci., 249, 729-739.

von Huene, R., Nasu, N., Fujioka, K., et al., 1978. Japan Trench transected. Geotimes, 23, 16-21.

Wager, L. R., and Deer, W. A., 1939. The petrology of the Skaergaard intrusion, Kangerdlugssuaq, east Greenland. Med. Groen., 105 (No. 4).

Westgate, J. A., and Fulton, R. J., 1975. Tephrostratigraphy of Olympia interglacial sediments in south-central British Columbia, Canada. Can. J. Earth Sci., 12, 489-502.

Yokoyama, T., 1972. Discrimination of the volcanic ash layers by means of the features of the volcanic glass, with special reference to the difference of the titanium contents in the volcanic glass of the Plio-Pleistocene Osaka Group, Japan. Jpn. Assoc. Quat. Res., 11, 247-253.

Yoshikawa, S., 1978. Chemical composition of glass in volcanic ash layers of the Osaka Group. J. Geol. Soc. Jpn., $84,131-140$. 